\title{
Polarization lidar for detecting dust orientation: System design and calibration.
}

\author{
Alexandra Tsekeri ${ }^{1}$, Vassilis Amiridis ${ }^{1}$, Alexandros Louridas ${ }^{2}$, George Georgoussis ${ }^{2}$, \\ Volker Freudenthaler ${ }^{3}$, Spiros Metallinos ${ }^{1}$, George Doxastakis ${ }^{2}$, Josef Gasteiger ${ }^{4}$, Nikolaos Siomos ${ }^{1}$, \\ Peristera Paschou ${ }^{1}$, Thanasis Georgiou ${ }^{1}$, George Tsaknakis ${ }^{2}$, Christos Evangelatos ${ }^{2}$, and \\ Ioannis Binietoglou ${ }^{5}$ \\ ${ }^{1}$ Institute for Astronomy, Astrophysics, Space Applications and Remote Sensing, National Observatory of Athens, Athens, \\ Greece \\ ${ }^{2}$ Raymetrics S.A., Athens, Greece \\ ${ }^{3}$ Fakultät für Physik, Meteorologisches Institut, Ludwig-Maximilians-Universität, Munich, Germany \\ ${ }^{4}$ University of Vienna, Faculty of Physics, Vienna, Austria \\ ${ }^{5}$ National Institute of R\&D for Optoelectronics, Magurele, Romania
}

Correspondence: Alexandra Tsekeri (atsekeri@noa.gr)

\begin{abstract}
Dust orientation is an ongoing investigation in recent years. Its potential proof will be a paradigm shift for dust remote sensing, invalidating the currently used simplifications of randomly-oriented particles. Vertically-resolved measurements of dust orientation can be acquired with a polarization lidar designed to target the off-diagonal elements of the backscatter matrix which are non-zero only when the particles are oriented. Building on previous studies, we constructed a lidar system emitting linearly- and elliptically-polarized light at $1064 \mathrm{~nm}$ and detecting the linear and circular polarization of the backscattered light. Its measurements provide direct flags of dust orientation, as well as more detailed information of the particle microphysics. The system also employs the capability to acquire measurements at varying viewing angles. Moreover, in order to achieve good signal-to-noise-ratio in short measurement times the system is equipped with two laser sources emitting in interleaved fashion, and two telescopes for detecting the backscattered light from both lasers. Herein we provide a description of the optical and mechanical parts of this new lidar system, the scientific and technical objectives of its design, and the calibration methodologies tailored for the measurements of oriented dust particles. We also provide the first measurements of the system.
\end{abstract}

\section{Introduction}

Dust particles have non-spherical irregular shapes and they have been reported to present preferential orientation (Ulanowski et al., 2007). If present, particle orientation will play a role in the radiation reaching the surface of the Earth and the top of the atmosphere, as well as in the interpretation of the remote sensing observations used for dust monitoring from space, that cannot be described using the long-established assumption of randomly-oriented particles. The phenomenon of dust orientation has been extensively studied for space dust (e.g., Whitney and Wolff, 2002), whereas the investigation for the Earth's atmosphere is a relatively new field of research. Specifically, the only signature of dust orientation in the Earth's atmosphere comes from astronomical polarimetry measurements of dichroic extinction during a dust event at the Canary islands (Ulanowski et al., 
2007) and a dust event at the Antikythera island in Greece (Daskalopoulou et al., 2021). However these measurements refer to column-integrated values, not being capable for vertically-resolved retrievals.

Lidars (light detection and ranging) are capable of providing vertically-resolved measurements of dust orientation in the atmosphere. Previous studies have demonstrated the feasibility of using circularly- or linearly-polarized lidar measurements to detect the orientation of ice crystals in clouds (Kaul et al., 2004; Hayman et al., 2012; Balin et al., 2013; Volkov et al., 2015; Kokhanenko et al., 2020) and it has been theoretically shown that these techniques can be extended to oriented dust particles (Geier and Arienti, 2014). Specifically, Geier and Arienti (2014) demonstrated that although the linearly-polarized measurements provided by most of the lidar systems are sufficient for discerning ice crystal orientation, this is not the case for smaller particles as dust, for which we expect much lower differences than the order(s) of magnitude reported for oriented particles in clouds. What they suggested is to use light that is linearly-polarized along a plane at an angle $\neq 0$, or circularlypolarized light, and detect the backscattered light at different polarization planes. With this approach they showed that the off-diagonal elements of the backscatter phase matrix could be retrieved, providing information on the orientation of the particles.

In this work we propose a different approach for the polarization lidar we designed and constructed, aiming for direct measurements of dust orientation, without having to retrieve the individual off-diagonal elements of the backscatter phase matrix, and also aiming at sufficient information on dust microphysical properties. The new lidar, nicknamed WALL-E, employs two lasers, with the first emitting linearly-polarized light along a plane at $45^{\circ}$ with respect to the horizon, and the second emitting elliptically-polarized light, with the angle of the polarization ellipse at $5.6^{\circ}$ with respect to the horizon. The two laser sources emit interleavingly and their backscattered light is collected also interleavingly, for both, by two telescopes. At the detection side, after the first telescope, the linear polarization of the backscattered light is measured, whereas after the second telescope the circular polarization of the backscattered light is measured. The operating wavelength is at $1064 \mathrm{~nm}$ for better probing the dust coarse mode. The system is capable of measuring at more than one zenith and azimuth viewing angles so as to provide more information on the dust orientation and microphysical properties, depending on the angle of the particle orientation. In order to derive the orientation properties of the particles with respect to the horizon, we define the polarization of the emitted and detected light with respect to the horizon. To achieve highly-accurate polarization measurements with high signal-to-noise ratio (SNR), the lidar system uses high-power lasers, large aperture telescopes and small receiver field-of-view. For the design and calibration of the system we followed the high quality standards of the European Aerosol Research Lidar Network (EARLINET) (Freudenthaler, 2016).

In Section 2 we present a general description of the instrument, including mainly the optical and the mechanical parts of the system. In Section 3 we describe the methodology we followed for the system design, based on specific scientific and technical objectives. In Sections 4 and 5 we present the various calibration procedures. In Section 6 we provide a methodology for comparing the measurements with the ones from commonly-used polarization lidars, using the volume linear depolarization ratio. In Section 7 we present the first measurements of the system, acquired during a dust case in Athens, Greece. In Section 8 we provide an overview of this work and we discuss its future perspectives. The detailed calculations for the methodologies 
presented herein are provided in the Appendices A, B and C, as well as in the Supplement. Moreover, a table containing all acronyms and symbols is also provided in the Supplement.

\section{Overview of the lidar components}

The lidar system is equipped with two emission units, and two detection units. The lasers in the emission units emit interleaved light pulses, and their backscattered light is measured interleavingly for each laser at the detectors of the detection units. Each of the detection units is comprised of a telescope, polarizing optics and two detectors. The system uses this "two-laser/twotelescope/four-detector" setup to record eight separate signals.

The lidar has been developed by Raymetrics S.A. and it is housed in a compact enclosure that permits the system to perform measurements in the field, under a wide range of ambient conditions. As shown in Fig. 1, it is comprised by the upper "head" part, containing the lasers, the telescopes and the detection units of the system, the bottom "electronics compartment", containing the power supplies of the lasers, the Transient Recorders (TRs), the Master Trigger Control Unit and the Lidar Peripheral Controlling unit (LPC), and the "positioner" which holds the head and facilitates its movement along different zenith and azimuth angles. The electronics compartment and the head are connected with two umbilical tubes that contain the cooling lines of the lasers and the power and communication cables.

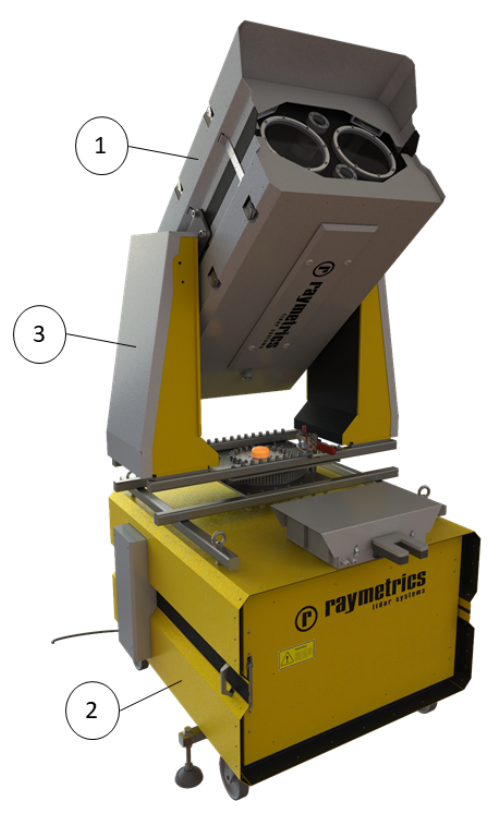

Figure 1. The lidar system, with the "head" part at the top (1), the "electronics compartment" at the bottom (2), and the "positioner" of the head (3). 


\subsection{The head}

The head of the system contains the lasers, the telescopes, and the rest of the detection units. The lasers and telescopes are placed in a diamond-shaped layout (Fig. 2, right), that ensures equal distances of both lasers from both telescopes, for the proper alignment of the laser beams with the field-of-view of both telescopes.

We use Nd:Yag lasers (CFR400 from Lumibird S.A.), emitting at $1064 \mathrm{~nm}$, with energy per pulse of $\sim 250 \mathrm{~mJ}$ (Table 1). We expand the laser beams by 5 times with beam expanders of Galilean type. Each laser and beam expander are mounted on a metallic plate which ensures their alignment and proper expanding of the outgoing expanded laser beam. In front of the lasers we place appropriate optical elements in order to change the polarization state of the emitted light, as described in Section 3. Specifically, in front of the "laser A" we place a Half Wave Plate $(H W P)$ to change the plane of its linear polarization to the plane at $45^{\circ}$ with respect to the horizon, and in front of the "laser B" we place a Quarter Wave Plate $(Q W P)$ followed by a $H W P$, to change its linear polarization to elliptical polarization, with the angle of the polarization ellipse at $5.6^{\circ}$ with respect to the horizon, and degree of linear polarization of 0.866 . The $Q W P$ and the $H W P s$ are mounted on stepping-index rotational mounts (with accuracy of $0.1^{\circ}$ ), which enable us to accurately rotate them to the desired positions and produce the desired polarization states, as described in Section 4.

The telescopes are of Dall-Kirkham type, with an aperture of $200 \mathrm{~mm}$ and focal length of $1000 \mathrm{~mm}$ (F\#5). The full overlap of the laser beams to the telescope field-of-views is achieved above $400-600 \mathrm{~m}$.

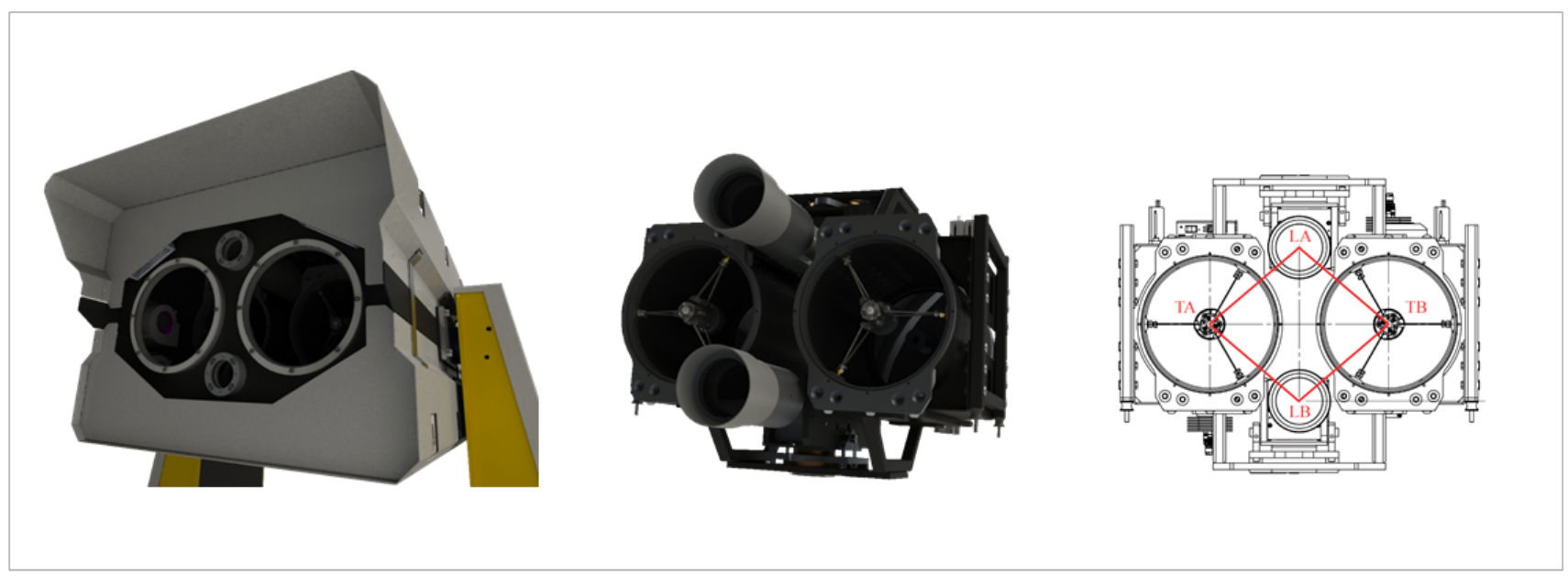

Figure 2. The lidar head. Left: Cover of the lidar head, showing the windows in front of the two lasers and the two telescopes. Middle: Photorealistic image of the internal parts of the head, showing the lasers and their beam expanders, the telescopes and the rest of the detection units. Right: Front view of the head, showing the diamond-shaped layout of the lasers (LA and LB) and telescopes (TA and TB). 
https://doi.org/10.5194/amt-2021-30

Preprint. Discussion started: 15 February 2021

(c) Author(s) 2021. CC BY 4.0 License.

(c) (i)

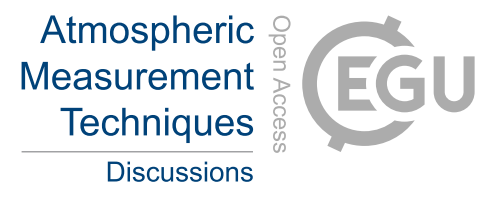

Table 1. Laser A and B parameters

\begin{tabular}{lcc} 
& laser A & laser B \\
\hline Nominal wavelength (nm) & 1064 & 1064 \\
\hline Energy (mJ) & 256.1 & 256.9 \\
\hline Near Field Beam Diameter (mm) & 6.25 & 6.14 \\
\hline Pulse Width - FWHM (nsec) & 7.29 & 7.46 \\
\hline Divergence at 86.5\% (mrad) & 1.11 & 0.73 \\
\hline Pulse Rate (Hz) & 20 & 20
\end{tabular}

The detection units after the telescopes (Fig. 3) contain the optical elements (e.g. $H W P, Q W P$, Polarizing Beam Splitter cube $P B S$ ) that alter the Stokes vector of the collected backscattered light, so as to measure its polarization state effectively, as discussed in detail in Section 3. The signals are recorded by two cooled Avalanche PhotoDiodes (APDs) at each detection unit, which contain remote-controlled power supplies and cooling units. Each detection unit contains also a shutter for performing dark measurements, a camera for the alignment of the lasers with the telescopes, and an interference filter for reducing the background light in the measurements. 


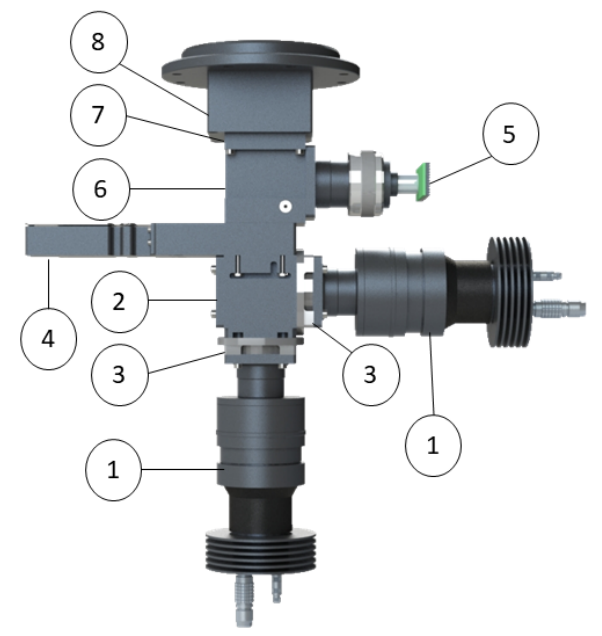

Figure 3. The detection unit after telescope A, containing the optical elements that are used for the detection of the polarization state of the backscattered light, with the signals recorded at the two APDs (1). It also contains a $P B S$ cube (2), followed by cleaning polarizing sheet filters (3), a shutter for dark measurements (4), a camera for the alignment (5), a turning mirror for redirecting the light to the camera (6), an interference filter for the reduction of the background light (7), and a mechanical rotator (8) for accurately rotating a $H W P$ for the system calibration (Section 4). The detection unit after telescope B is the same, with a $Q W P$ placed before the $P B S$. telescopes (Fig. 2, left). The covers can be easily removed to allow access to the internal parts of the head. Moreover, thermoelectric coolers are installed inside the head in order to stabilize the internal temperature, and to provide tolerance to ambient temperatures up to $45^{\circ} \mathrm{C}$.

\subsection{The positioner}

95 The positioner enables the lidar head to move along different zenith and azimuth angles. Due to constrains from the umbilical tubes, the head can be moved along $-10^{\circ}$ to $+90^{\circ}$ from the zenith, and at $-150^{\circ}$ to $+150^{\circ}$ around the vertical. The positioner consists of two side arms and a base (Fig. 4) which can be manually rotated. For changing the zenith angle, the one of the side arms is driving and the other is free. A break on the free arm reduces the backlash. 
https://doi.org/10.5194/amt-2021-30

Preprint. Discussion started: 15 February 2021

(c) Author(s) 2021. CC BY 4.0 License.
Atmospheric

Measurement

Techniques

Discussions

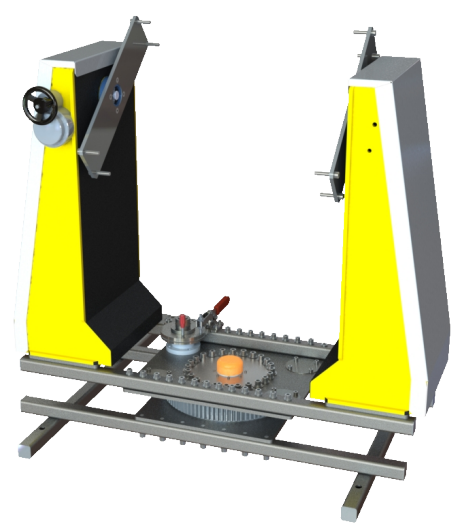

Figure 4. The fork-type positioner of the lidar head.

\subsection{The electronics compartment}

The electronics compartment (Fig. 5) contains the power supplies of the two lasers, the LPC, the LICEL rack containing the TRs for digitizing and recording the signals from the APDs, and the Master Trigger Control Unit that synchronizes the emission of the two lasers and the acquisition of the backscattered signals. Moreover, it contains a UPS and a precipitation sensor.

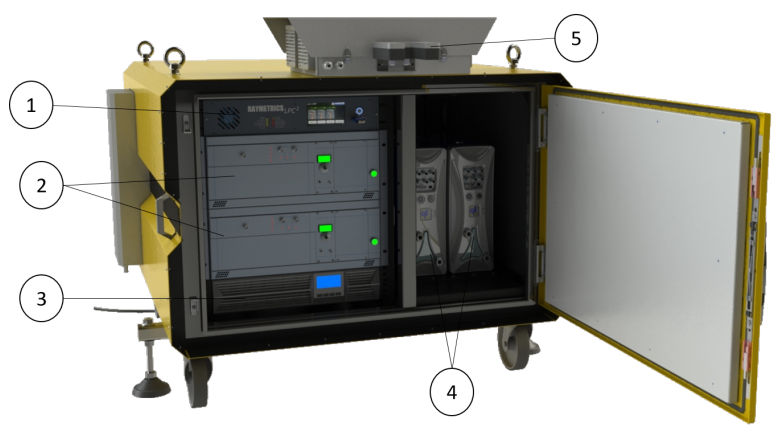

Figure 5. The enclosure with 1) the LPC unit, 2) the LICEL rack with the TRs and the Master Trigger Control, 3) the UPS, 4) the power supplies of the lasers, and 5) the precipitation sensor.

The synchronization of this complicated lidar system with two lasers emitting interleavingly and with their backscattered signals recorded interleavingly, requires a sophisticated triggering system. We use a master trigger control unit, produced by Licel GmbH (Fig. 6) that utilizes two trigger generators for the synchronization of the emission of the lasers and of the 
acquisition of the signals. As shown in Fig. 6b, the first trigger generator produces a pulse that starts the flash lamp. The laser builds up its maximum energy for $160 \mu \mathrm{sec}$, and then a second pulse turns on the active Q-switch, which allows the release of the laser beam pulse. In the meantime, a third pulse triggers the acquisition of the backscattered light from laser A. Pre-trigger measurements are acquired until the emission of the laser A beam pulse. The same sequence is performed from the second trigger generator for laser B, starting $10 \mathrm{~ms}$ later, in order to avoid the recording of overlapping photons from the backscattered light of laser A. The duty cycle of lasers A and B is $\sim 12 \mathrm{~ms}$ and the time between each cycle is $50 \mathrm{~ms}$ (fixed due to the repetition rate of the lasers of $20 \mathrm{~Hz}$ ), with $38 \mathrm{~ms}$ idle time.

a)

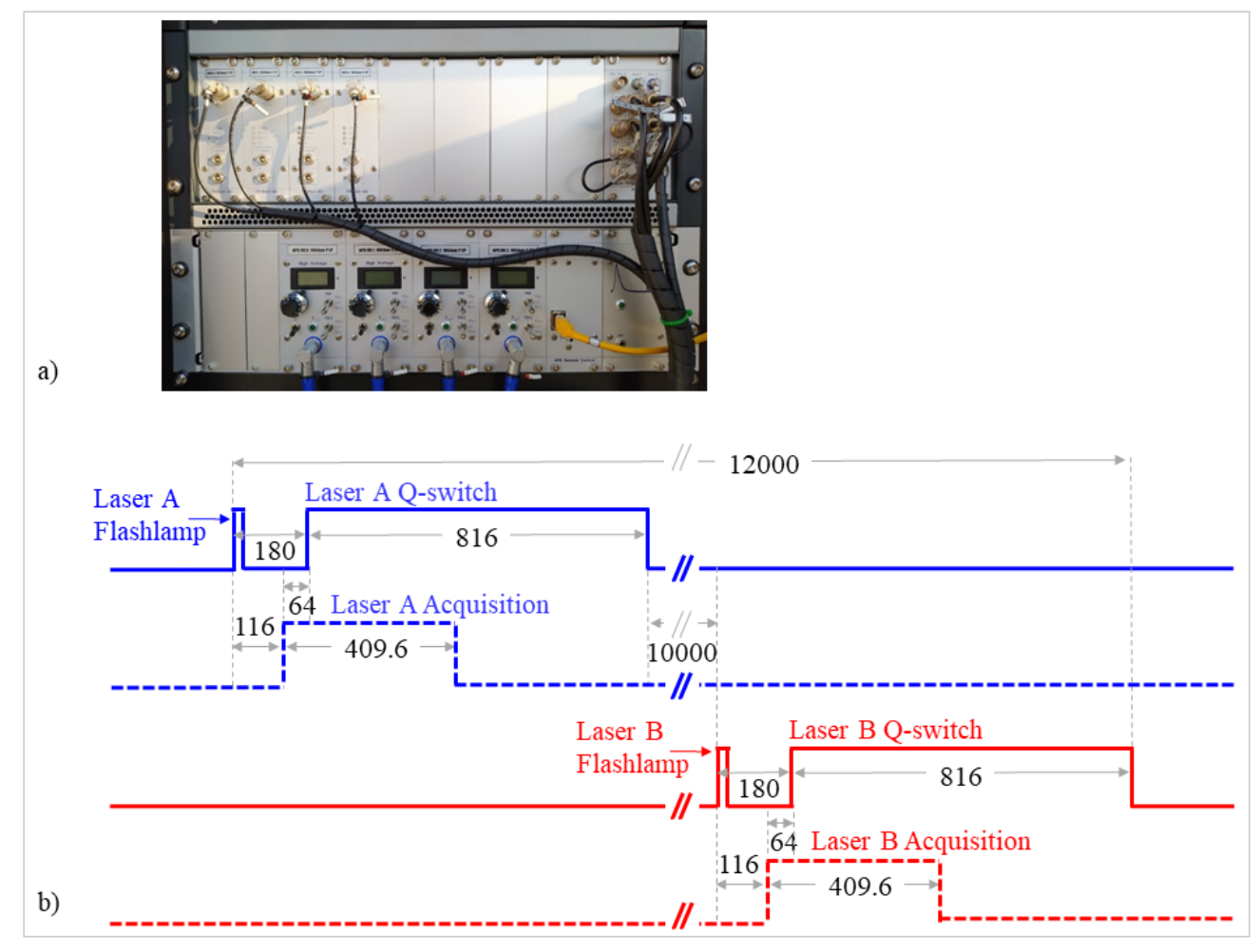

Figure 6. a) The LICEL rack with the TRs and the Master Trigger Control Unit. b) The pulses from the Master Trigger Control Unit that synchronize the emission of the two lasers and the acquisition of the backscattered signals. The lengths of the pulses are in $\mu$.

The lidar system is controlled from the LPC unit. This is an "enhanced" embedded computer with specific I/Os that fits the lidar requirements, providing several ethernet interfaces that makes the controlling (local or remote) of the lidar easy and safe. Temperature and humidity conditions inside the enclosure and the head can be recorder with the LPC. Additionally, the precipitation sensor (Fig. 5) provides information about precipitation conditions. Moreover, several hardware interlocks are 
connected to the LPC and shut down the lasers in case of emergency. The LPC also controls the mechanical rotators of the optical elements used for calibration purposes (Section 4).

\section{Emission and detection design based on the measurement strategy}

The core of the new lidar system is its emission and detection design, based on our measurement strategy for monitoring the oriented dust in the atmosphere. Our approach is different from the measurement strategy of previous works, which either focus on the retrieval of the individual elements of the backscatter matrix of the oriented particles utilizing moving elements in the system (e.g., Kaul et al., 2004), or use complicated designs that are difficult to be calibrated effectively (e.g., Geier and Arienti, 2014). We choose to avoid both, in order to achieve robust measurements along with their effective calibration. Moreover, most of the previous works utilize visible light measurements whereas we use near infrared light measurements at $1064 \mathrm{~nm}$, to better probe the larger dust particles (a more detailed discussion is provided in Tsekeri et al. (2021)).

Figure 7 shows the simplest design of a "two lasers/two telescopes/four detectors" lidar system that is able to detect the elliptically-polarized backscattered light from oriented particles in the atmosphere without using any moving parts. The linear polarization of the backscattered signal is detected using a linear-polarization-analyzer (a $P B S$ ) in the detection unit after telescope $\mathrm{A}$, and the circular polarization of the backscattered signal is detected using a circular-polarization-analyzer (a $Q W P$ followed by a $P B S$ ) in the detection unit after telescope $\mathrm{B}$. The calibration methodology is based on the solutions introduced by Freudenthaler (2016) for EARLINET lidar systems, as well as on new methodologies tailored for the detection of oriented particles, presented in Section 4.

Instead of retrieving the individual off-diagonal elements of the backscatter matrix $\mathbf{F}$ (Eq. 1), we aim for measurements that are combinations of only the off-diagonal elements of the backscatter matrix that will be nonzero only in case of oriented particles. This way we acquire direct measurements of dust orientation, in the form of flags of "yes" or "no" particle orientation. This first-level information of the oriented dust in the atmosphere is straightforward to achieve, since it does not require any inversion procedure. Moreover, it is important to have, considering that the phenomenon of dust orientation has not been extensively observed in the Earth's atmosphere, even at this elementary level. To achieve this, laser A should emit linearlypolarized light at $45^{\circ}$, as discussed in detail below.

$\mathbf{F}=\left[\begin{array}{cccc}F_{11} & F_{12} & F_{13} & F_{14} \\ F_{12} & F_{22} & F_{23} & F_{24} \\ -F_{13} & -F_{23} & F_{33} & F_{34} \\ F_{14} & F_{24} & -F_{34} & F_{44}\end{array}\right]=F_{11}\left[\begin{array}{cccc}1 & f_{12} & f_{13} & f_{14} \\ f_{12} & f_{22} & f_{23} & f_{24} \\ -f_{13} & -f_{23} & f_{33} & f_{34} \\ f_{14} & f_{24} & -f_{34} & f_{44}\end{array}\right]$

Where, $f_{i j}=\frac{F_{i j}}{F_{11}}$. 
https://doi.org/10.5194/amt-2021-30

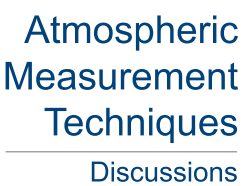

Preprint. Discussion started: 15 February 2021

(c) Author(s) 2021. CC BY 4.0 License.

(c) (1)

Discussions

Along with the measurements of "orientation flags", we aim for measurements that provide additional information for the particle orientation properties, as the particle orientation angle and the percentage of oriented particles in the atmosphere, as well as information on dust microphysics, i.e. an estimation of the particle size and refractive index. These are parameters that are necessary to have in order to explain the phenomenon of dust orientation in more detail. The methodology for defining the optimum measurements includes simulated atmospheric scenarios and machine learning tools, and is presented in Tsekeri et al. (2021). Briefly, the backscattered light is simulated for different orientation angles and mixtures of random and oriented particles, as well as irregular shapes and realistic sizes for dust particles. In order to derive signals that provide additional information for the dust orientation and microphysics, the light of laser B should be elliptically-polarized so as the backscattered signals contain more elements of the backscatter matrix. 

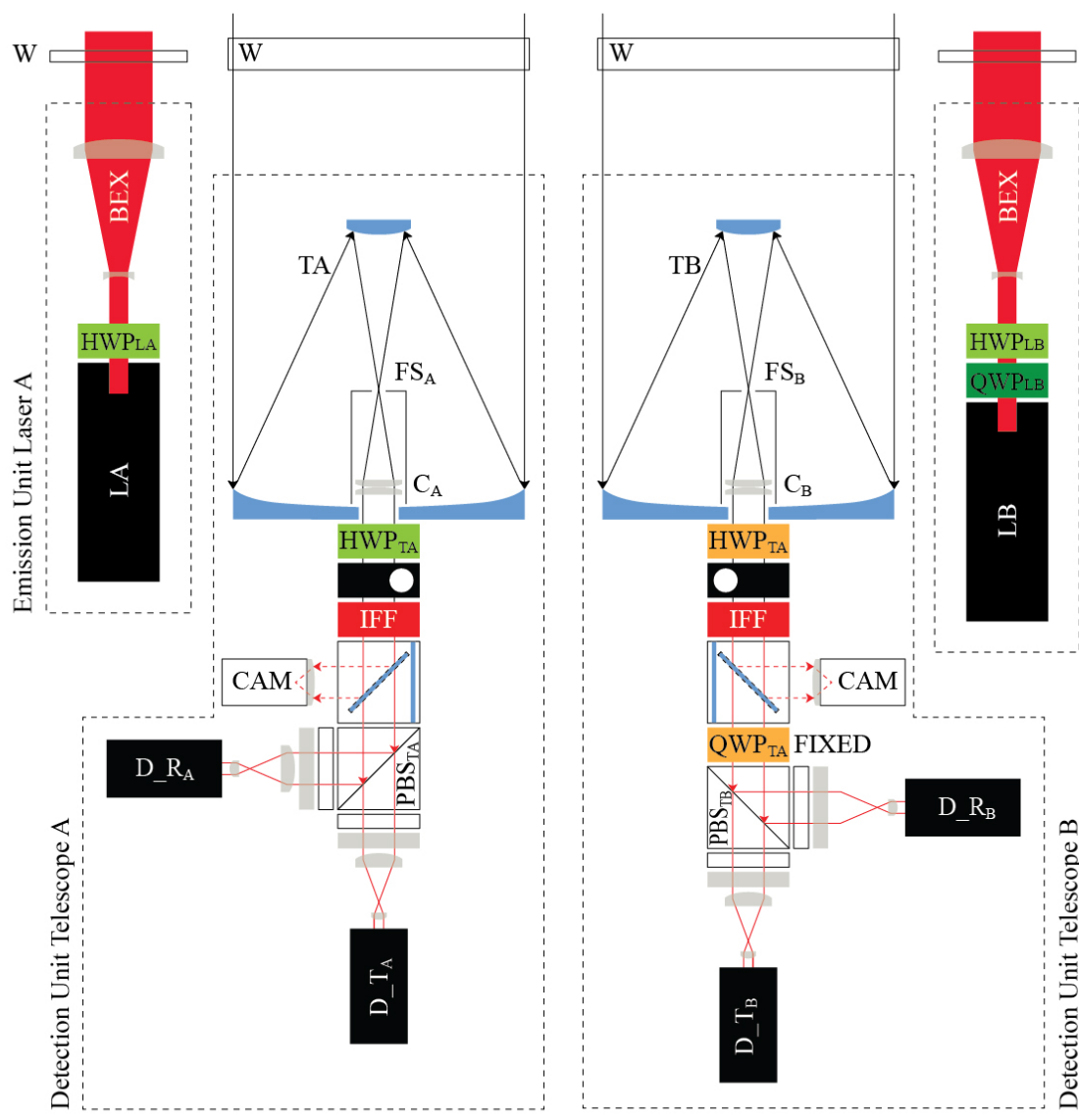

Figure 7. Sketch of the system design: two lasers shooting alternatively ( $L_{A}$ and $L_{B}$ ), with the backscattered signals correspondingly alternatively collected by two telescopes $\left(T_{A}\right.$ and $\left.T_{B}\right)$ and then redirected at two detectors for each telescope $\left(D \_S_{j}, S=T, R\right.$ as of "Transmitted" and "Reflected" channels, $j=A, B$ ). The polarization of the light emitted from each laser is changed appropriately, using the $H W P_{L A}$ for laser A and the $Q W P_{L B}$ followed by the $H W P_{L B}$ for laser B. The laser beam of each laser is expanded with a beam expander (BEX). After the first telescope the light goes through $P B S_{T A}$ and after the second telescope the light goes through $Q W P_{T B}$ and $P B S_{T B}$. The $H W P_{T A}$ at telescope A is used to correct the rotation of the $P B S_{T A}$ (Section 4.1). The $H W P_{T B}$ at telescope B is used to check the position of the $Q W P_{T B}$ with respect to the $P B S_{T B}$ (Section S4 in Supplement)). The shutter at each telescope is used for performing dark measurements. The camera at each telescope is used for the alignment of the laser beams with the field-of-view of the telescope.

This Section provides the methodology we followed to define the properties of the optical elements in the emission and the detection units of the lidar system, so as to fulfil the technical and scientific objectives of our measurement strategy. Considering the layout in Fig. 7 the only "free" parameters that we need to define are the polarization state of the light from the emission 
units of laser $\mathrm{A}$ and $\mathrm{B}$, and the position of the $Q W P_{T B}$ in the detection unit after telescope $\mathrm{B}$ (the $H W P_{T A}$ and $H W P_{T B}$ are used for calibration purposes (see Section 4 and Section S4 in the Supplement)).

The measured signal $I_{i_{-} k_{-} S}^{*}$ for laser $i=L A, L B$, at the detection unit after telescope $k=T A, T B$, at the detector $S=T$, $R$ ("Transmitted" and "Reflected" channel after the $P B S_{k}$, respectively), is shown in Eq. 2-4.

$I_{i_{-} k_{-} S}^{*}=E_{i_{-} k} \eta_{S_{-} k} \boldsymbol{e} \mathbf{M}_{k}\left[(\mathbf{F}+\mathbf{G}) \boldsymbol{i}_{i}+\boldsymbol{i}_{g}\right]+y_{N_{i_{-} k_{-} S}}$

$\mathbf{M}_{T B}=\mathbf{M}_{S_{-} T B} \mathbf{M}_{Q W_{-} T B} \mathbf{M}_{O_{-} T B}$

In Eq. 2, $E_{i_{\_} k}=A_{k} O_{i_{\_} k} T(0, r)^{-2} E_{o i}$, where $A_{k}$ is the area of the telescope $k, O_{i \_k}$ is the overlap function of the laser beam receiver field-of-view with range 0-1 (for laser $i$ and telescope $k$ ), $T(0, r)$ is the transmission of the atmosphere between the lidar at range $r=0$ and a specific range in the atmosphere, and $E_{o i}$ is the pulse energy of laser $i . \eta_{S_{-} k}$ is the amplification of the signals at $S=T$ or $R$ detector of the detection unit after telescope $k . e=[1,0,0,0]^{T}$ is used to select the first component of the Stokes vector, which corresponds to the signal intensity measured at the APDs. $\mathbf{M}_{k}$ is the Mueller matrix of the detection unit after telescope A (Eq. 3) and B (Eq. 4): $\mathbf{M}_{S_{-} k}$ is the Mueller matrix of the $P B S_{k}$ followed by cleaning polarizing sheet filters, $\mathbf{M}_{O_{-} k}$ is the Mueller matrix of the receiver optics (i.e. telescope $k$, collimating lenses, bandpass filter), and $\mathbf{M}_{Q W_{-} T B}$ is the Mueller matrix of the $Q W P_{T B} . \mathbf{F}$ and $\mathbf{G}$ are the backscatter Stokes phase matrices of the dust particles and of the gas molecules, respectively, at a certain range in the atmosphere. For the explicit definition of the Muller matrices in Eq. 2, 3 and 4, see Section S1 in the Supplement. $\boldsymbol{i}_{i}$ is the Stokes vector of the light from the emission unit of laser $i$, and $\boldsymbol{i}_{g}$ is the Stokes vector of the background skylight. $y_{N_{i_{-} k_{-} S}}$ is the electronic background of the $S=T$ or $R$ detector at the detection unit after telescope $k$, for detecting the backscattered signal of laser $i$.

After subtracting the background skylight signal offset, and considering noise-free measurements, the signals $I_{i_{-}} T A_{-} S$ and $I_{i_{-} T B_{-} S}$, are provided in Eq. 5 and 6, respectively.

$I_{i_{-} T A \_S}=E_{i_{-} T A} \eta_{S_{-} T A} \boldsymbol{e} \mathbf{M}_{S_{-} T A} \mathbf{M}_{O_{-} T A}(\mathbf{F}+\mathbf{G}) \boldsymbol{i}_{i}$

$$
I_{i_{-} T B \_S}=E_{i_{-} T B} \eta_{S_{-} T B} \boldsymbol{e} \mathbf{M}_{S_{-} T B} \mathbf{M}_{Q W_{-} T B} \mathbf{M}_{O_{-} T B}(\mathbf{F}+\mathbf{G}) \boldsymbol{i}_{i}
$$


Performing the Mueller matrix calculations, $I_{i_{-} T A_{-} S}$ and $I_{i_{-} T B_{-} S}$ can be written as a function of the Stokes parameters of the light from the emission units of the lasers $\boldsymbol{i}_{\mathbf{i}}=\left[I_{i}, Q_{i}, U_{i}, V_{i}\right]^{T}$, the fast-axis-angle $\phi_{T B}$ of the $Q W P_{T B}$ with the reference plane, and the backscatter Stokes phase matrix elements (all highlighted in Eq. 7 and 8). In Eq. 7 and 8 we consider ideal receiver optics (i.e. telescope, collimating lenses, bandpass filter), with no diattenuation, retardation and misalignment effects.

$$
\begin{aligned}
& \frac{I_{i_{-} T A_{-} S}}{E_{i_{-} T A} \eta_{S_{-} T A} T_{S_{-} T A} T_{O_{-} T A} \boldsymbol{F}_{\mathbf{1 1}}}=\boldsymbol{I}_{\boldsymbol{i}}+\left(D_{S_{-} T A} \boldsymbol{I}_{\boldsymbol{i}}+\boldsymbol{Q}_{\boldsymbol{i}}\right) \boldsymbol{f}_{\mathbf{1 2}}+\boldsymbol{U}_{\boldsymbol{i}} \boldsymbol{f}_{\mathbf{1 3}}+\boldsymbol{V}_{\boldsymbol{i}} \boldsymbol{f}_{\mathbf{1 4}}+D_{S_{-} T A} \boldsymbol{Q}_{\boldsymbol{i}} \boldsymbol{f}_{\mathbf{2 2}}+ \\
& +D_{S_{-} T A} \boldsymbol{U}_{\boldsymbol{i}} \boldsymbol{f}_{\mathbf{2 3}}+D_{S_{-} T A} \boldsymbol{V}_{\boldsymbol{i}} \boldsymbol{f}_{\mathbf{2 4}}+\boldsymbol{I}_{\boldsymbol{i}} g_{11}+D_{S_{-} T A} \boldsymbol{Q}_{\boldsymbol{i}} g_{22} \\
& \frac{I_{i_{-} T B_{-} S}}{E_{i_{-} T B} \eta_{S_{-} T B} T_{S_{-} T B} T_{O_{-} T B} \boldsymbol{F}_{\mathbf{1 1}}}=\boldsymbol{I}_{\boldsymbol{i}}+\left(D_{S_{-} T B} c_{2 \phi_{\boldsymbol{T} B}}^{2} \boldsymbol{I}_{\boldsymbol{i}}+\boldsymbol{Q}_{\boldsymbol{i}}\right) \boldsymbol{f}_{\mathbf{1 2}}+\left(-D_{S_{-} T B} c_{2 \phi_{\boldsymbol{T} B}} s_{2 \phi_{\boldsymbol{T} B}} \boldsymbol{I}_{\boldsymbol{i}}+\boldsymbol{U}_{\boldsymbol{i}}\right) \boldsymbol{f}_{\mathbf{1 3}}+ \\
& +\left(-D_{S_{-} T B} s_{2 \phi_{T B}} \boldsymbol{I}_{\boldsymbol{i}}+\boldsymbol{V}_{\boldsymbol{i}}\right) \boldsymbol{f}_{\mathbf{1 4}}+D_{S_{-} T B} c_{2 \phi_{T B}}^{2} \boldsymbol{Q}_{\boldsymbol{i}} \boldsymbol{f}_{\mathbf{2 2}}+ \\
& +D_{S_{-} T B}\left(-c_{2 \phi_{T B}} s_{2 \phi_{T B}} \boldsymbol{Q}_{\boldsymbol{i}}+c_{2 \boldsymbol{\phi}_{T B}}^{2} \boldsymbol{U}_{\boldsymbol{i}}\right) \boldsymbol{f}_{\mathbf{2 3}}+D_{S_{-} T B}\left(-s_{2 \phi_{\boldsymbol{T} B}} \boldsymbol{Q}_{\boldsymbol{i}}+c_{2 \boldsymbol{\phi}_{\boldsymbol{T} B}}^{2} \boldsymbol{V}_{\boldsymbol{i}}\right) \boldsymbol{f}_{\mathbf{2 4}}+ \\
& +D_{S_{-} T B} c_{2 \phi_{T B}} s_{2 \phi_{T B}} \boldsymbol{U}_{\boldsymbol{i}} \boldsymbol{f}_{\mathbf{3 3}}+D_{S_{-} T B}\left(s_{2 \phi_{T B}} \boldsymbol{U}_{\boldsymbol{i}}+c_{2 \phi_{T B}} s_{2 \phi_{T B}} \boldsymbol{V}_{\boldsymbol{i}}\right) \boldsymbol{f}_{\mathbf{3 4}}-D_{S_{-} T B} s_{2 \phi_{T B}} \boldsymbol{V}_{\boldsymbol{i}} \boldsymbol{f}_{\mathbf{4 4}}+ \\
& +\boldsymbol{I}_{\boldsymbol{i}} g_{11}+D_{S_{-} T B} c_{2 \phi_{\boldsymbol{T} B}}^{2} \boldsymbol{Q}_{\boldsymbol{i}} g_{22}+D_{S_{-} T B} c_{2 \phi_{T B}} s_{2 \phi_{T B}} \boldsymbol{U}_{\boldsymbol{i}} g_{33}-D_{S_{-} T B} s_{2 \phi_{T B}} \boldsymbol{V}_{\boldsymbol{i}} g_{44}
\end{aligned}
$$

Where, $g_{i j}=\frac{G_{i j}}{F_{11}}, c_{2 \phi_{T B}}=\cos \left(2 \phi_{T B}\right), s_{2 \phi_{T B}}=\sin \left(2 \phi_{T B}\right), T_{S_{-} k}$ is the unpolarized transmittance $(S=T)$ or reflectance $(S=R)$ of the $P B S_{k}$, and $D_{S_{-} k}$ is the diattenuation parameter of the transmitted or reflected channels of the $P B S_{k}$ followed by the cleaning polarizing sheet filters $\left(D_{T_{-} k}=1\right.$ and $D_{R_{-} k}=-1$, respectively, see Section S1 in Supplement).

As can be deduced by Eq. 7 and 8, in order to use laser A to achieve measurements that contain only the off-diagonal elements of the backscatter matrix, the following conditions must be met: $Q_{L A}=0$ for $I_{L A_{-} T A_{-} S}, V_{L A}=0$ for $I_{L A_{-} T B_{-} S}$, and $\phi_{T B}=45^{\circ}$. Thus, the Stokes vector of the light from the emission unit of laser A should be $45^{\circ}$-linearly polarized, $\boldsymbol{i}_{\mathbf{L A}}=[1,0,1,0]^{T}$. This is achieved using the $H W P_{L A}$ in front of laser A (Fig. 7), as discussed in Section 4.2. Moreover, the fast-axis-angle of the $Q W P_{T B}$ should be at $\phi_{T B}=45^{\circ}$.

For calibration reasons, we use the ratios of the measurements of the reflected and transmitted channels after the $P B S_{k}$ (Eq. 9 and 10). The calibrated backscatter signal ratios of laser A provide a direct flag of particle orientation $\left(F_{L A \_T A}\right.$ and $F_{L A \_T B}$ in Eq. 12 and 13, respectively), when their values are $\neq 1$.

$\frac{I_{L A \_T A \_R}}{I_{L A \_} T A_{-} T}=\eta_{T A} \frac{1-\boldsymbol{f}_{\mathbf{1 2}}+\boldsymbol{f}_{\mathbf{1 3}}-\boldsymbol{f}_{\mathbf{2 3}}+g_{11}}{1+\boldsymbol{f}_{\mathbf{1 2}}+\boldsymbol{f}_{\mathbf{1 3}}+\boldsymbol{f}_{\mathbf{2 3}}+g_{11}}$

$\frac{I_{L A \_T B_{-} R}}{I_{L A_{-} T B_{-} T}}=\eta_{T B} \frac{1+\boldsymbol{f}_{\mathbf{1 3}}+\boldsymbol{f}_{\mathbf{1 4}}-\boldsymbol{f}_{\mathbf{3 4}}+g_{11}}{1+\boldsymbol{f}_{\mathbf{1 3}}-\boldsymbol{f}_{\mathbf{1 4}}+\boldsymbol{f}_{\mathbf{3 4}}+g_{11}}$ 
The calibration factors $\eta_{T A}=\frac{\eta_{R_{-} T A} T_{R_{T A} A}}{\eta_{T_{-} T A} T_{T_{-} T A}}$ and $\eta_{T B}=\frac{\eta_{R_{T} T B} T_{R_{-} T B}}{\eta_{T_{-} T B} T_{T_{-} T B}}$ are derived as shown in Section 5 .

Due to the $H W P_{T B}$ in the detection unit after telescope B (used for checking for systematic errors in measurements, as discussed in Section S4 in the Supplement)), Eq. 10 changes to Eq. 11.

$\frac{I_{L A \_T B \_R}}{I_{L A \_T B_{-} T}}=\eta_{T B} \frac{1+\boldsymbol{f}_{\mathbf{1 3}}-\boldsymbol{f}_{\mathbf{1 4}}+\boldsymbol{f}_{\mathbf{3 4}}+g_{11}}{1+\boldsymbol{f}_{\mathbf{1 3}}+\boldsymbol{f}_{\mathbf{1 4}}-\boldsymbol{f}_{\mathbf{3 4}}+g_{11}}$

The orientation flags $F_{L A \_} T A$ and $F_{L A \_T B}$ are provided in Eq. 12 and 13.

$F_{L A \_T A}=\frac{1}{\eta_{T A}} \frac{I_{L A \_T A \_R}}{I_{L A \_} T A_{-} T}$

$F_{L A \_T B}=\frac{1}{\eta_{T B}} \frac{I_{L A \_T B \_R}}{I_{L A \_T B \_}}$

Having achieved the orientation flags with laser A, we use laser B to increase the information content of the measurements in terms of dust orientation properties (e.g. angle and percentage of oriented particles in the atmosphere) and of dust microphysical properties (e.g. size and refractive index). For this reason, the light from the emission unit of laser B is elliptically-polarized with the angle of the polarization ellipse at $5.6^{\circ}$ and Stokes vector $\boldsymbol{i}_{\mathrm{LB}}=[1,0.85,0.17,0.5]^{T}$. The derivation of the optimum polarization state of $\boldsymbol{i}_{\mathrm{LB}}$ is provided in detail in Tsekeri et al. (2021), using an extended simulated dataset for various atmospheric scenes with oriented dust particles. In their work, the backscattered light is simulated for different orientation angles and mixtures of random and oriented particles, as well as irregular shapes and realistic sizes for dust particles. The elliptical polarization of $\boldsymbol{i}_{\mathrm{LB}}$ is set with the $Q W P_{L B}$ and the $H W P_{L B}$ in front of laser B (Fig. 7), as discussed in Section 4.3. The corresponding signal ratios are shown in Eq. 14 and 15.

$\frac{I_{L B \_} T A_{-} R}{I_{L B \_} A_{-} T}=\eta_{T A} \frac{1+0.15 \boldsymbol{f}_{\mathbf{1 2}}+0.17 \boldsymbol{f}_{\mathbf{1 3}}+0.5 \boldsymbol{f}_{\mathbf{1 4}}-0.85 \boldsymbol{f}_{\mathbf{2 2}}-0.17 \boldsymbol{f}_{\mathbf{2 3}}-0.5 \boldsymbol{f}_{\mathbf{2 4}}+g_{11}-0.85 g_{22}}{1+1.85 \boldsymbol{f}_{\mathbf{1 2}}+0.17 \boldsymbol{f}_{\mathbf{1 3}}+0.5 \boldsymbol{f}_{\mathbf{1 4}}+0.85 \boldsymbol{f}_{\mathbf{2 2}}+0.17 \boldsymbol{f}_{\mathbf{2 3}}+0.5 \boldsymbol{f}_{\mathbf{2 4}}+g_{11}+0.85 g_{22}}$

$\frac{I_{L B_{-} T B_{-} R}}{I_{L B_{-} T B_{-} T}}=\eta_{T B} \frac{1+0.85 \boldsymbol{f}_{\mathbf{1 2}}+0.17 \boldsymbol{f}_{\mathbf{1 3}}+1.5 \boldsymbol{f}_{\mathbf{1 4}}+0.85 \boldsymbol{f}_{\mathbf{2 4}}-0.17 \boldsymbol{f}_{\mathbf{3 4}}+0.5 \boldsymbol{f}_{\mathbf{4 4}}+g_{11}+0.5 g_{44}}{1+0.85 \boldsymbol{f}_{\mathbf{1 2}}+0.17 \boldsymbol{f}_{\mathbf{1 3}}-0.5 \boldsymbol{f}_{\mathbf{1 4}}-0.85 \boldsymbol{f}_{\mathbf{2 4}}+0.17 \boldsymbol{f}_{\mathbf{3 4}}-0.5 \boldsymbol{f}_{\mathbf{4 4}}+g_{11}-0.5 g_{44}}$ 16. 
$\frac{I_{L B_{-} T B_{-} R}}{I_{L B_{-} T B_{-} T}}=\eta_{T B} \frac{1+0.85 \boldsymbol{f}_{\mathbf{1 2}}+0.17 \boldsymbol{f}_{\mathbf{1 3}}-0.5 \boldsymbol{f}_{\mathbf{1 4}}-0.85 \boldsymbol{f}_{\mathbf{2 4}}+0.17 \boldsymbol{f}_{\mathbf{3 4}}-0.5 \boldsymbol{f}_{\mathbf{4 4}}+g_{11}-0.5 g_{44}}{1+0.85 \boldsymbol{f}_{\mathbf{1 2}}+0.17 \boldsymbol{f}_{\mathbf{1 3}}+1.5 \boldsymbol{f}_{\mathbf{1 4}}+0.85 \boldsymbol{f}_{\mathbf{2 4}}-0.17 \boldsymbol{f}_{\mathbf{3 4}}+0.5 \boldsymbol{f}_{\mathbf{4 4}}+g_{11}+0.5 g_{44}}$

\section{Definition of the polarization of the emitted and detected light with respect to the horizon}

The polarization of the light emitted and detected by the system should be defined with respect to the horizon, so as the retrieved properties of the oriented particles are defined with respect to the horizon. This is done by first leveling the head of the lidar along the horizon using a spirit level, which then enables us to use the frame of the lidar head as the reference coordinate system. The "frame coordinate system" (Fig. 8a) is a right-handed coordinate system, with $x_{F}$-axis parallel to the horizon and the $z_{F}$-axis pointing in propagation direction of the emitted light from lasers $\mathrm{A}$ and $\mathrm{B}$, considering that both lasers are parallel.

The optical elements are considered to be perfectly aligned with eachother in the detection units after telescopes A and B (because their holders are manufactured and assembled in a mechanical workshop with high accuracy), but the detection units are possibly rotated around the optical axis with respect to the frame coordinate system by angles $\omega_{T A}$ and $\omega_{T B}$, respectively (Fig. $8 \mathrm{~b}$ and c). The Stokes vectors of the light collected at telescope A and B are consequently described including a multiplication with the rotation matrices $\mathbf{R}_{\mathbf{T A}}\left(-\omega_{T A}\right)$ and $\mathbf{R}_{\mathbf{T B}}\left(-\omega_{T B}\right)$, respectively (see Eq. S.5.1.7 in Freudenthaler (2016)), which affects the measurements of the polarized components after $P B S_{T A}$, but not after $P B S_{T B}$, as shown in Appendix A.

The rotation of the detection unit after telescope A is corrected using the $H W P_{T A}$, as shown in Section 4.1.

The " $D U_{T A}$ coordinate system" and the " $D U_{T B}$ coordinate system" in Fig. $8 \mathrm{~b}$ and c are the right-handed coordinate systems of the detection units after telescopes A and B, respectively. The $x_{D U_{T A}}$ and $y_{D U_{T A}}$ axis coincide with the incidence plane of $P B S_{T A}$, and the $x_{D U_{T B}}$ and $y_{D U_{T B}}$ axis coincide with the incidence plane of $P B S_{T B}$.

Equations A1-A6 in Appendix A show the formulation of Eq. 7 and 8 for $I_{i} k_{-} S$ with respect to the frame coordinate system, taking into account all the optical elements of the system, along with the rotation of the detection units after telescopes A and B. The analytic derivations of Eq. A1-A6 are provided in Section S2 of the Supplement. 

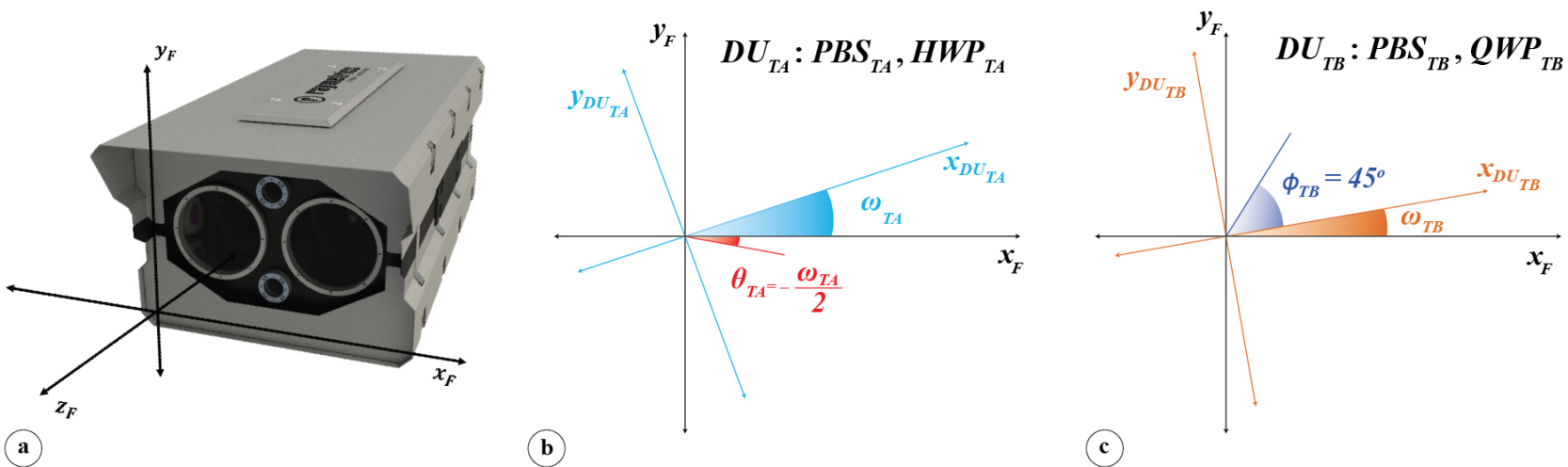

(b)

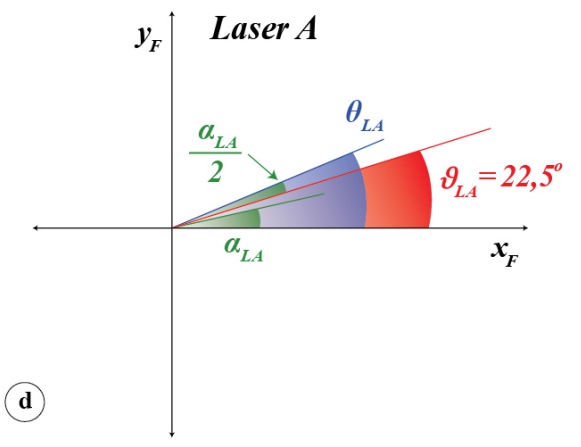

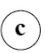

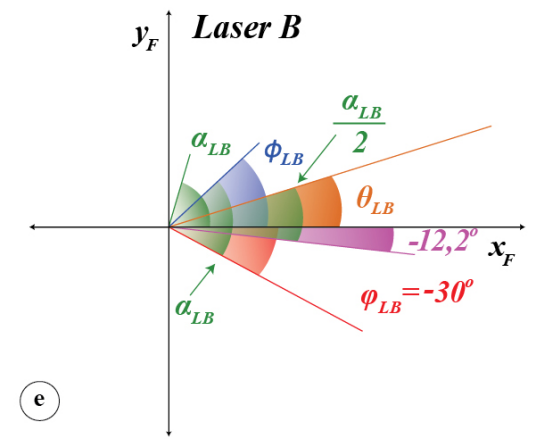

Figure 8. a) The "frame coordinate system" (black) is the reference coordinate system with $x_{F}$-axis parallel to the horizon. b) The " $D U_{T A}$ coordinate system" (light blue) is the coordinate system of the detection unit after telescope A, which is rotated with respect to the frame coordinate system by an angle $\omega_{T A}$. The effect of this rotation on the signals is corrected using $H W P_{T A}$, placed at $\theta_{T A}=-\frac{\omega_{T A}}{2}$ (red) with respect to the $x_{F}$-axis. c) The "DU $U_{T B}$ coordinate system" (orange) is the coordinate system of the detection unit after telescope $\mathrm{B}$, which is rotated with respect to the frame coordinate system by an angle $\omega_{T B}$. The rotation does not affect the measured signals. The $Q W P_{T B}$ before $P B S_{T B}$, is placed at $\phi_{T B}=45^{\circ}$ with respect to the $x_{D U_{T B}}$-axis. d) The light emitted directly from laser A is linearly-polarized with unknown angle of polarization $\alpha_{L A}$. As shown in Eq. 17, using the $H W P_{L A}$ with fast-axis-angle $\theta_{L A}=22.5^{\circ}+\frac{\alpha_{L A}}{2}$, we produce the light emitted from the emission unit of laser A with angle of polarization $2 \vartheta_{L A}=45^{\circ}$. e) The light emitted directly from laser B is linearlypolarized with unknown angle of polarization $\alpha_{L B}$. As shown in Eq. 18, using the $Q W P_{L B}$ with with fast-axis-angle $\phi_{L B}=\alpha_{L B}-30^{\circ}$, and the $H W P_{L B}$ with fast-axis-angle $\theta_{L B}=\frac{\alpha_{L B}}{2}-12.2^{\circ}$, we produce the elliptically-polarized light emitted from the emission unit of laser B with angle of polarization $5.6^{\circ}$ and degree of linear polarization 0.866 .

The Stokes vector of the light from the emission unit of laser A and B is provided by $\boldsymbol{i}_{L A}(\mathrm{Eq} .17)$ and $\boldsymbol{i}_{L B}(\mathrm{Eq} .18$ ), respectively. The light emitted directly from laser A $\left(\boldsymbol{i}_{l s r_{-} L A}\right)$ and laser B $\left(\boldsymbol{i}_{l s r_{-} L B}\right)$ is considered to be $100 \%$ linearly-polarized, with angle of polarization ellipse with respect to the frame coordinate system $\alpha_{L A}$ and $\alpha_{L B}$, respectively, i.e. $\boldsymbol{i}_{l s r_{-} L A}\left(\alpha_{L A}\right)=$ $\left[\begin{array}{llll}1 & c_{2 \alpha_{L A}} & s_{2 \alpha_{L A}} & 0\end{array}\right]^{T}$ in Eq. 17, and $\boldsymbol{i}_{l s r_{-} L B}\left(\alpha_{L B}\right)=\left[\begin{array}{llll}1 & c_{2 \alpha_{L B}} & s_{2 \alpha_{L B}} & 0\end{array}\right]^{T}$ in Eq. 18. The angles $\alpha_{L A}$ and $\alpha_{L B}$ are 
unknown a-priori. The polarization of the light from the whole emission unit is defined according to the position of the optical elements in front of the lasers with respect to the frame coordinate system, i.e. the fast-axis-angle $\theta_{L A}$ of the $H W P_{L A}$ in front of laser A, and the fast-axis-angle $\phi_{L B}$ of $Q W P_{L B}$ followed by the $H W P_{L B}$ with fast-axis-angle $\theta_{L B}$ in front of laser B (Fig. $8 \mathrm{~d}$ and e; Eq. 17 and 18).

In order to simplify Eq. 17 and 18 we use the angles $\vartheta_{L A}=\theta_{L A}-\frac{\alpha_{L A}}{2}$ and $\varphi_{L B}=\phi_{L B}-\alpha_{L B}$. From Eq. 17 and 18 we deduce: $\vartheta_{L A}=22.5^{\circ}$ and $\theta_{L A}=22.5^{\circ}-\frac{\alpha_{L A}}{2}$ (Fig. 8d), $\varphi_{L B}=-30^{\circ}, \phi_{L B}=\alpha_{L B}-30^{\circ}$ and $\theta_{L B}=\frac{\alpha_{L B}}{2}-12.2^{\circ}$ (Fig. 8e).

$\boldsymbol{i}_{L A}=\mathbf{M}_{H W_{-} L A}\left(\theta_{L A}\right) \boldsymbol{i}_{l s r_{-} L A}\left(\alpha_{L A}\right)=\left[\begin{array}{c}1 \\ c_{\left(4 \theta_{L A}-2 \alpha_{L A}\right)} \\ s_{\left(4 \theta_{L A}-2 \alpha_{L A}\right)} \\ 0\end{array}\right]=\left[\begin{array}{c}1 \\ c_{4 \vartheta_{L A}} \\ s_{4 \vartheta_{L A}} \\ 0\end{array}\right]=\left[\begin{array}{l}1 \\ 0 \\ 1 \\ 0\end{array}\right]$

$\boldsymbol{i}_{L B}=\mathbf{M}_{H W_{-} L B}\left(\theta_{L B}\right) \mathbf{M}_{Q W_{-} L B}\left(\phi_{L B}\right) \boldsymbol{i}_{l s r_{-} L B}\left(\alpha_{L B}\right)=\left[\begin{array}{c}1 \\ c_{2\left(\phi_{L B}-\alpha_{L B}\right)} c_{\left(4 \theta_{L B}-2 \phi_{L B}\right)} \\ c_{2\left(\phi_{L B}-\alpha_{L B}\right)} s_{\left(4 \theta_{L B}-2 \phi_{L B}\right)} \\ -s_{2\left(\phi_{L B}-\alpha_{L B}\right)}\end{array}\right]=\left[\begin{array}{c}1 \\ c_{2 \varphi_{L B}} c_{\left(4 \theta_{L B}-2 \phi_{L B}\right)} \\ c_{2 \varphi_{L B}} s_{\left(4 \theta_{L B}-2 \phi_{L B}\right)} \\ -s_{2 \varphi_{L B}}\end{array}\right]=\left[\begin{array}{c}1 \\ 0.85 \\ 0.17 \\ 0.5\end{array}\right]$

\subsection{Correction of the signal $I_{i_{-} T A}{ }_{-} S$, due to the rotation of the detection unit after telescope $A$}

Equations $\mathrm{A} 1$ and $\mathrm{A} 3$ in Appendix A show that the rotation of the detection unit after telescope A changes the signals $I_{L A} T A_{-} S$ (Eq. 9) and $I_{L B_{-} T A_{-} S}$ (Eq. 14), respectively. In order to correct for this effect, we have to set the fast-axis-angle of the $H W P_{T A}$ at $\theta_{T A}=-\frac{\omega_{T A}}{2}$ with respect to the $x_{D U_{T A}}$-axis (Fig. 8b), so that $c_{\left(4 \theta_{T A}+2 \omega_{T A}\right)}=1$ and $s_{\left(4 \theta_{T A}+2 \omega_{T A}\right)}=0$ in Eq. A1 and A3.

Since the value of $\theta_{T A}$ with respect to the $x_{D U_{T A}}$-axis is unknown a priori, we assume that it deviates from the desired value by an unknown angle $\varepsilon_{T A}$, thus $\theta_{T A}=\frac{-\omega_{T A}}{2}+\varepsilon_{T A}$. We derive $\varepsilon_{T A}$ by using the measurements from laser A, after placing a linear polarizer in front of the window of laser A at $45^{\circ}$ from $x_{F}$-axis (Fig. 9), and rotating the $H W P_{T A}$ in order to perform a methodology similar to the " $\Delta 90^{\circ}$ calibration" of Freudenthaler (2016), as in Fig. 10 and described in detail in Appendix B. The methodology is applicable only when there are only randomly-oriented particles in the atmosphere. 
https://doi.org/10.5194/amt-2021-30

Preprint. Discussion started: 15 February 2021

(c) Author(s) 2021. CC BY 4.0 License.

(c) (i)

\section{Atmospheric Measurement Techniques Discussions}

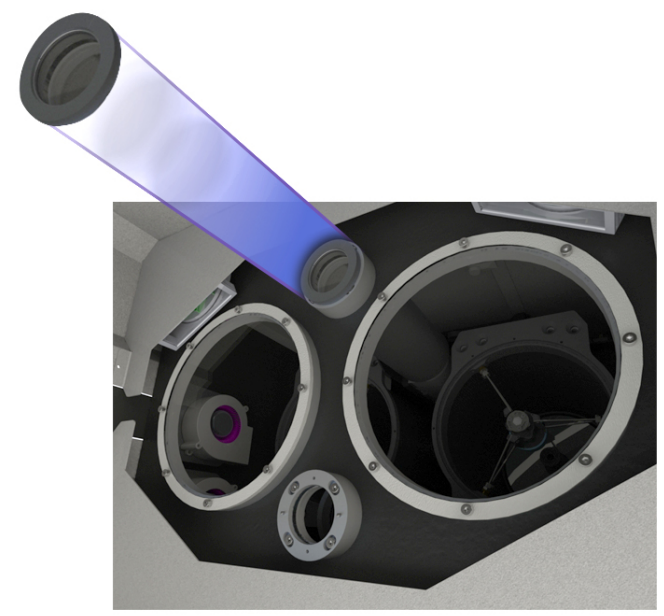

Figure 9. Linear polarizer in front of the window of laser A, placed at $45^{\circ}$ from $x_{F}$-axis. 

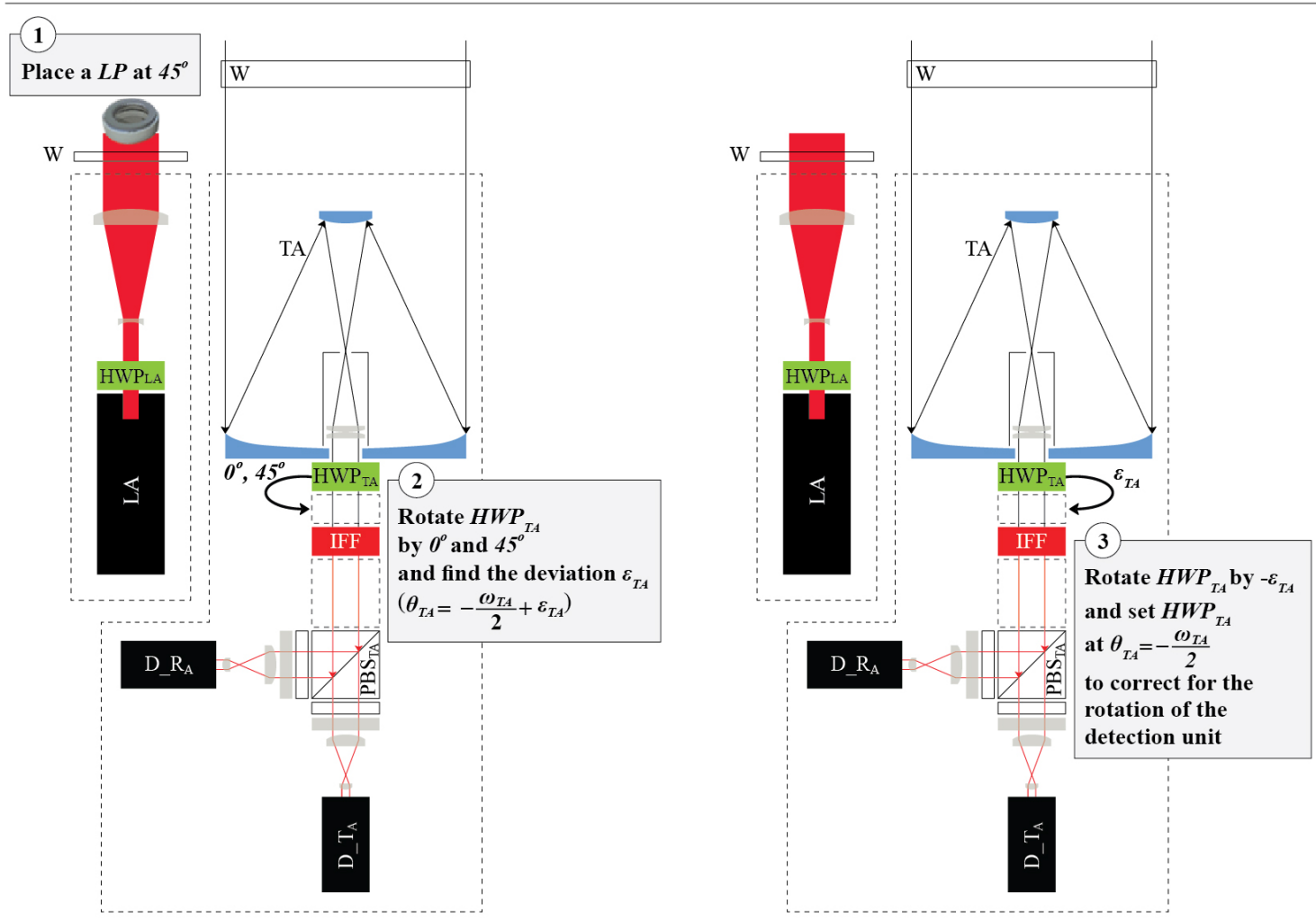

Figure 10. Methodology for correcting the measurements $I_{L i \_T A_{-} S}$ due to the rotation of the detection unit after telescope A.

\subsection{Definition of the polarization of the light from the emission unit of laser A with respect to the horizon}

In order to set the linear polarization of the light from the emission unit of laser $\mathrm{A}$ at $45^{\circ}$-degrees with respect to the horizon, as discussed in Section 3, we have to set $\vartheta_{L A}=22.5^{\circ}$ with respect to $x_{F}$-axis (Eq. 17; Fig. 8d). Since the value of $\vartheta_{L A}$ is unknown a priori, we assume that it is equal to an unknown angle $\varepsilon_{L A}$. We derive $\varepsilon_{L A}$ by performing the " $\Delta 90^{\circ}$ calibration" of Freudenthaler (2016) by rotating the $H W P_{L A}$ in front of laser A, as shown in Fig. 11 and discussed in detail in Appendix C. Then, we rotate the $H W P_{L A}$ by $22.5^{\circ}-\varepsilon_{L A}$ and set $\vartheta_{L A}=22.5^{\circ}$. ( $\vartheta_{L A}=\theta_{L A}-\frac{\alpha_{L A}}{2}$ (Eq. 17), thus in order to change $\vartheta_{L A}$ by angle $x$, it is sufficient to rotate the $H W P_{L A}$ and change its fast-axis angle $\theta_{L A}$ by angle $x$.)

Our methodology considers that the atmosphere consists of only randomly-oriented particles and that we have corrected the effect of the rotation of the detection unit after telescope A on signals $I_{L i} T A_{-} S$ (Section 4.1). 


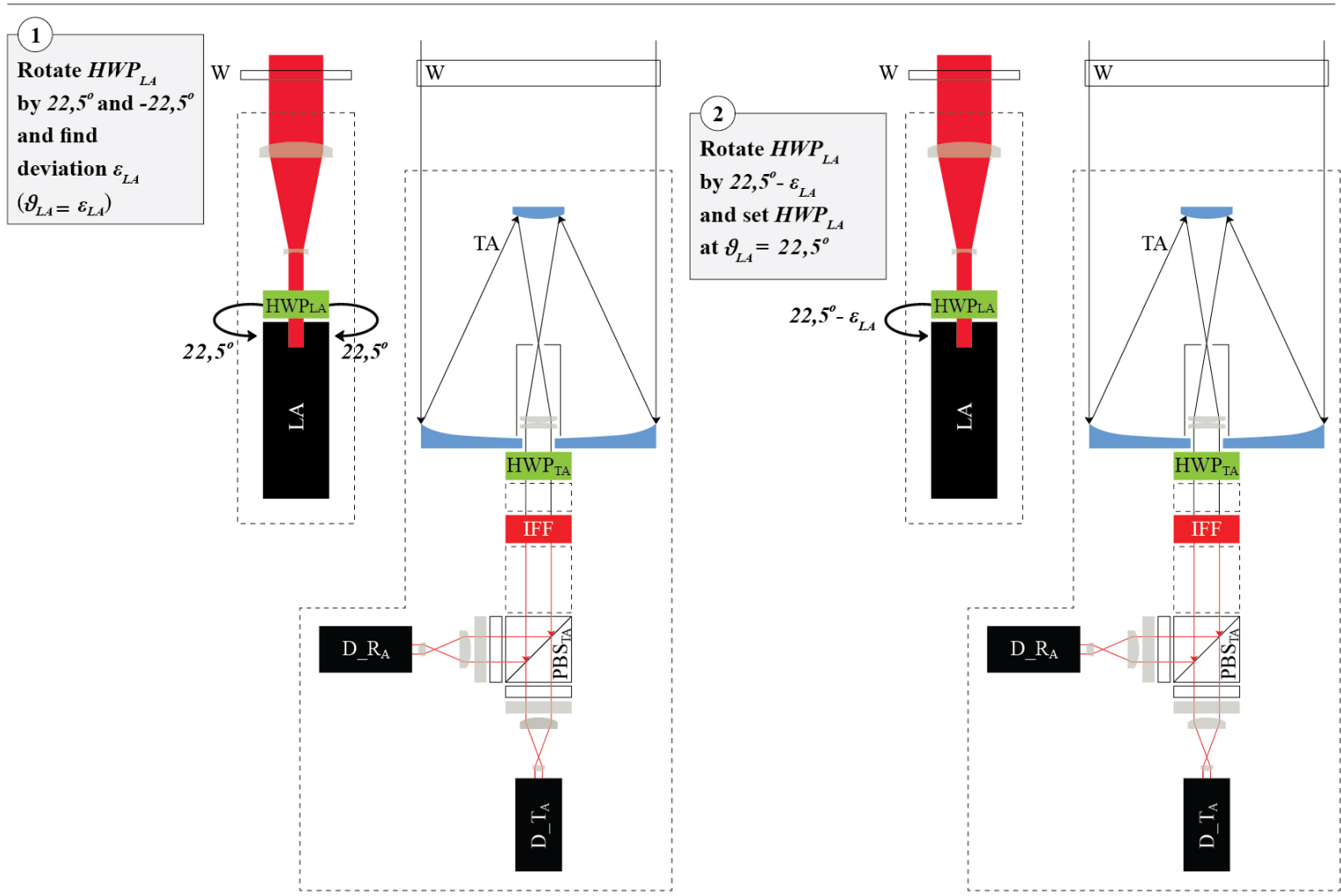

Figure 11. Methodology for defining the polarization of the light from the emission unit of laser A, with respect to the horizon.

\subsection{Definition of the polarization of the light from the emission unit of laser $B$ with respect to the horizon}

The desired elliptical polarization of the light from the emission unit of laser B with respect to the horizon is shown in Eq. 19, with angle of the polarization ellipse $\alpha_{e m}=5.6^{\circ}$ and degree of linear polarization $b_{e m}=0.866 . b_{e m}=c_{2 \varphi_{L B}}$, thus in order to set it to the desired value, we rotate the $Q W P_{L B}$ and change $\varphi_{L B}$ appropriately ( $\varphi_{L B}=\phi_{L B}-\alpha_{L B}$ (Eq. 18), thus for changing $\varphi_{L B}$ by angle $x$, it is sufficient to rotate the $Q W P_{L B}$ and change its fast-axis angle $\phi_{L B}$ by angle $x$.). After we have set $b_{e m}$, we set $\alpha_{e m}=2 \theta_{L B}-\phi_{L B}$ to the desired value, by rotating the $H W P_{L B}$ in front of laser $\mathrm{B}$. The angles $\alpha_{L B}, \varphi_{L B}$, $\phi_{L B}$ and $\theta_{L B}$ are shown in Fig. 8e.

Our methodology is described in Fig. 12. We consider randomly-oriented particles in the atmosphere and we use the measurements of laser B at the detection unit after telescope A. The detection unit is aligned with the frame coordinate system (Section 4.1). First, we consider that the polarization of the light from the emission unit of laser B with respect to the frame coordinate system is unknown, with unknown angle of the polarization ellipse $\alpha$ and degree of linear polarization $b$. In order to set them to $\alpha_{e m}, b_{e m}$, we perform the following steps: 
1. We derive $\alpha$ and $b$ by turning the $H W P_{L B}$ by appropriate angles, using a similar methodology to the " $\Delta 90^{\circ}$ calibration" of Freudenthaler (2016), as shown in Fig. 12, and in detail in Appendix D.

2. We change $\varphi_{L B}=\frac{a \cos (b)}{2}$ by turning the $Q W P_{L B}$ by $\frac{a \cos \left(b_{e m}\right)-a \cos (b)}{2}$. Then, $\varphi_{L B_{-} \text {new }}=\frac{a \cos \left(b_{e m}\right)}{2}$ and the degree of linear polarization is set to the desired value $b_{e m}$.

3. The turning of $Q W P_{L B}$ in (2), changes the angle of the polarization ellipse to an unknown value of $\alpha_{n e w}$. We calculate $\alpha_{n e w}$ using the methodology in (1).

4. After deriving $\alpha_{n e w}$, we set the angle of the polarization ellipse to the desired value $\alpha_{e m}$, by turning $H W P_{L B}$ by $\frac{\alpha_{e m}-\alpha_{n e w}}{2}$.

$\boldsymbol{i}_{L B}=\left[\begin{array}{c}1 \\ c_{2 \varphi_{L B}} c_{\left(4 \theta_{L B}-2 \phi_{L B}\right)} \\ c_{2 \varphi_{L B}} s_{\left(4 \theta_{L B}-2 \phi_{L B}\right)} \\ -s_{2 \varphi_{L B}}\end{array}\right]=\left[\begin{array}{c}1 \\ b_{e m} c_{2 \alpha_{e m}} \\ b_{e m} s_{2 \alpha_{e m}} \\ \sqrt{1-b_{e m}}\end{array}\right]=\left[\begin{array}{c}1 \\ 0.85 \\ 0.17 \\ 0.5\end{array}\right]$ 
https://doi.org/10.5194/amt-2021-30

Preprint. Discussion started: 15 February 2021

(c) Author(s) 2021. CC BY 4.0 License.
Atmospheric

Measurement

Techniques

Discussions

Use $H W P_{L B}$ and $Q W P_{L B}$ to define the polarization of laser B with respect to the horizon

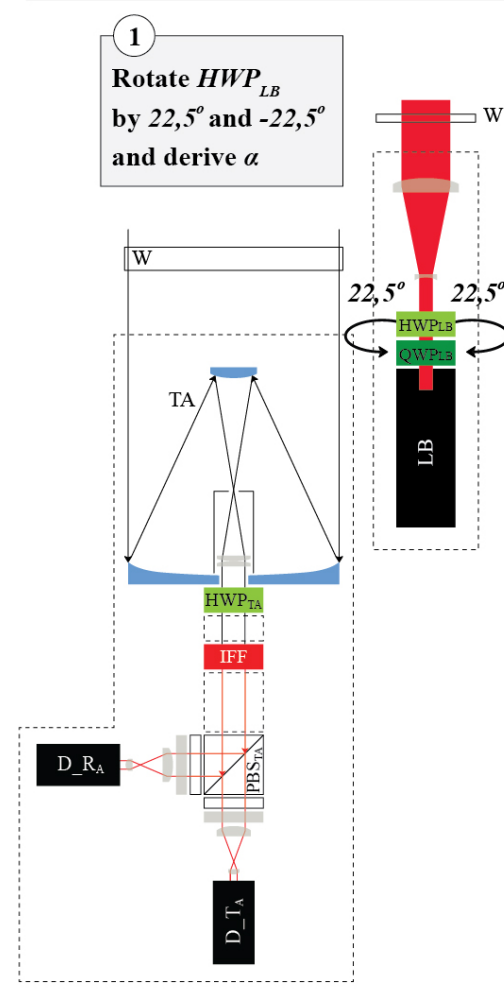

4

Rotate $\boldsymbol{H W P _ { L B }}$ by $22,5^{\circ}$ and $-22,5^{\circ}$ and derive $\alpha_{\text {new }}$

$\mathrm{D}_{-} \mathrm{R}_{\mathrm{A}}$

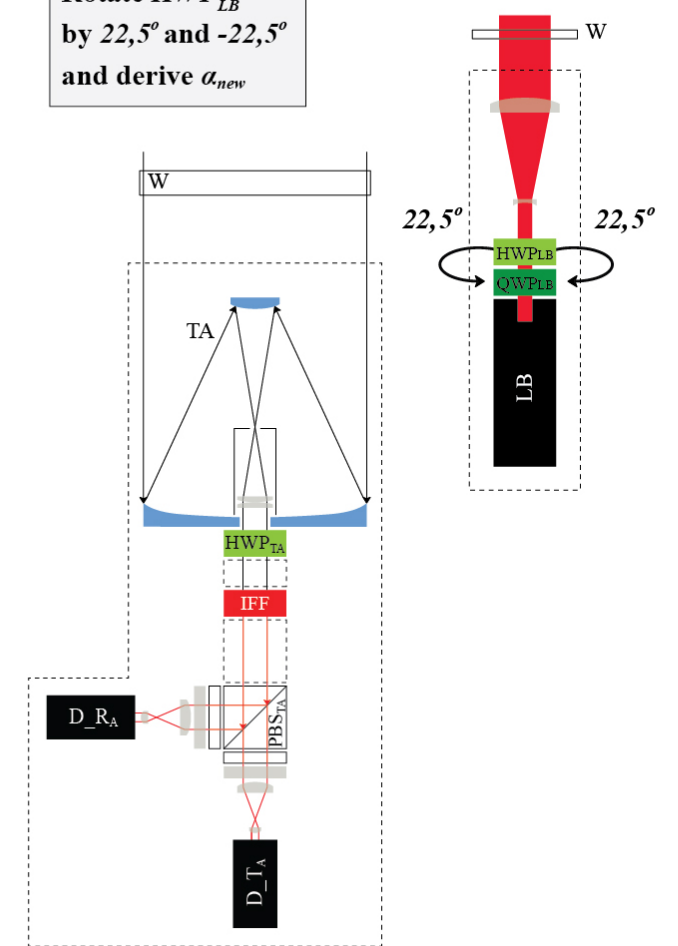

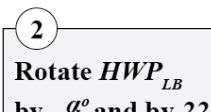

$$
\text { by }-\frac{\alpha^{o}}{2} \text { and by } 22,5^{\circ}-\frac{\alpha^{o}}{2}
$$

and derive $b$

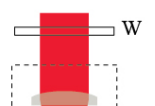

$\mathrm{D}_{-} \mathrm{R}_{\mathrm{A}}$

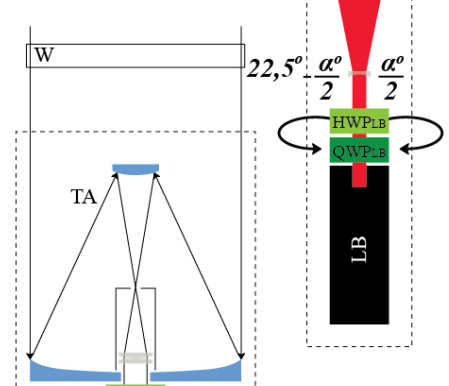

HWP

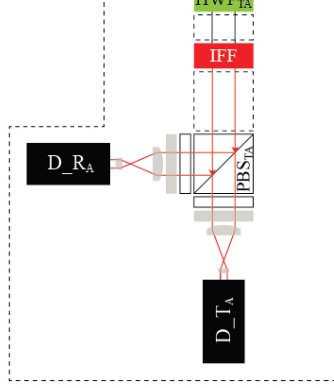

(3)

Rotate $Q W P_{L B}$ by $\frac{\alpha \cos \left(b_{e m}\right)-\alpha \cos (b)}{2}$

and set the degree of linear polarization

to the desired value $\boldsymbol{b}_{e m}$

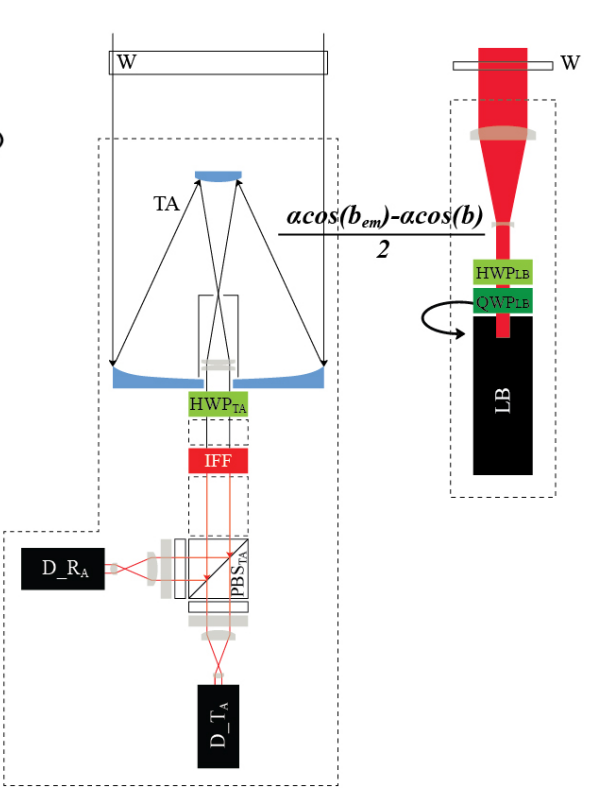

Rotate $\boldsymbol{H W P _ { L B }}$ by $\frac{\boldsymbol{\alpha}_{e m}-\boldsymbol{\alpha}_{\text {new }}}{2}$

and set the angle of polarization eclipse

to the desired value $a_{e m}$

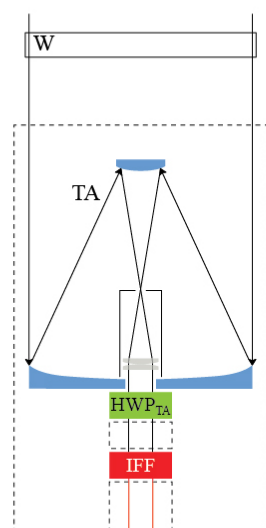

se

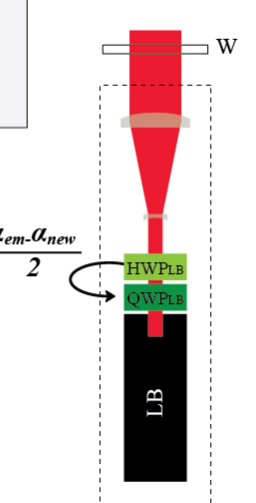

22

Figure 12. Methodology for defining the polarization of the emitted light from laser B with respect to the horizon. 


\section{The derivation of the calibration factors $\eta_{T A}$ and $\eta_{T B}$}

The calibration factors $\eta_{T A}$ and $\eta_{T B}$ are derived considering randomly-oriented particles in the atmosphere. We calculate $\eta_{T A}$ using the ratio of the signals from laser $\mathrm{A}$ at the detection unit after telescope A, considering that the effect of its rotation on the signals is corrected (Section 4.1). The $\eta_{T A}$ is calculated as shown in Eq. 20 (using Eq. A2 in Appendix A).

$\frac{I_{L A \_T A \_} R\left(\theta_{T A}=-\frac{\omega_{T A}}{2}\right)}{I_{L A \_T A \_T} T\left(\theta_{T A}=-\frac{\omega_{T A}}{2}\right)}=\frac{\eta_{R \_T A} T_{R \_T A}\left[f_{11}+g_{11}\right]}{\eta_{T \_} T A T_{T_{-} T A}\left[f_{11}+g_{11}\right]} \Rightarrow \eta_{T A}=\frac{I_{L A \_T A \_R\left(\theta_{T A}=-\frac{\omega_{T A}}{2}\right)}}{I_{L A \_T A \_T}\left(\theta_{T A}=-\frac{\omega_{T A} A}{2}\right)}$

We derive the calibration factor $\eta_{T B}$ using the ratio of the signals from laser $\mathrm{A}$ at the detection unit after telescope $\mathrm{B}$, as shown in Eq. 21 (using Eq. A5 in Appendix A).

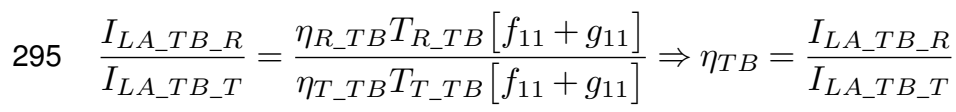

\section{The derivation of the volume linear depolarization ratio}

The volume linear depolarization ratio (VLDR) is a useful optical parameter for comparing the measurements of the new polarization lidar with measurements from the commonly-used polarization lidars, which emit linearly-polarized light and measure the corresponding cross- and parallel-polarized components of the backscattered light. The VLDR is calculated using the atmospheric polarization parameter $a$ as shown in Eq. 22.

$\delta=\frac{1-a}{1+a}$

We derive VLDR considering an atmosphere containing only randomly-oriented particles. Moreover, we consider that the effect of the rotation of the detection unit after telescope A with respect to the frame coordinate system is corrected (Section 4.1) and that the calibration factor $\eta_{T A}$ is calculated using measurements of laser A, as shown in Section 5.

We turn $H W P_{T A}$ by $22.5^{\circ}$, so as $\theta_{T A}=-\frac{\omega_{T A}}{2}+22.5^{\circ}$. Then, the measurements from laser $\mathrm{A}$ at the detection unit after telescope A are provided from Eq. 23, and the VLDR is calculated as shown in Eq. 24, using Eq. 22.

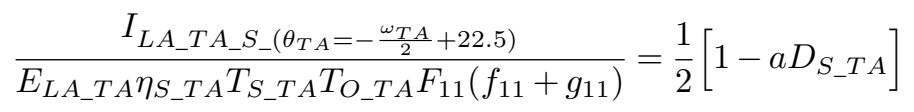


$\frac{I_{L A \_T A \_} \_\left(\theta_{T A}=-\frac{\omega_{T A}}{2}+22.5\right)}{I_{L A \_T A \_} A_{-}\left(\theta_{T A}=-\frac{\omega_{T A}}{2}+22.5\right)}=\eta_{T A} \frac{1+a}{1-a}=\frac{\eta_{T A}}{\delta} \Rightarrow \delta=\frac{1}{\eta_{T A}} \frac{I_{L A \_T A \_T}\left(\theta_{T A}=-\frac{\omega_{T A}}{2}+22.5\right)}{I_{L A \_T A \_} R_{-}\left(\theta_{T A}=-\frac{\omega_{T A}}{2}+22.5\right)}$

Due to the rotation of $H W P_{T A}$, the VLDR measurements cannot be acquired simultaneously with $F_{L A_{-} T A}$ (Eq. 12) and $\frac{I_{L B_{-} T A_{-} R}}{I_{L_{-} T A_{-} T}}$ (Eq. 14), but they are acquired simultaneously with $F_{L A_{-} T B}$ (Eq. 13) and $\frac{I_{L_{B} T B_{-} R}}{I_{L_{-} T B_{-} T}}$ (Eq. 16).

\section{First measurements}

We present measurements from Athens, Greece, on November 16, 2020, showing a dust layer. Figure 13 shows the 15-min averaged signals $I_{L A \_T A_{-} T}$ at $16: 22-18: 19$ UTC (Fig. 13a), with the corresponding orientation flags $F_{L A \_T A}$ (Eq. 12) shown in Fig. 13c. The orientation flag $F_{L A_{-} T B}$ (Eq. 13) was not measured, due to the removal of one of the APDs of the detection unit after telescope B on that day. The 15-min averaged signals $I_{L B_{-} T A_{-} T}$ at $16: 22-18: 19$ UTC are shown in Fig. 13b. The instrument points vertically. The minimum height of $600 \mathrm{~m}$ of the signals in the plots is the full-overlap height, derived by the telecover test (Freudenthaler et al., 2018).

The dust layer is at $1-2.5 \mathrm{~km}$, as shown in the VLDR profile in Fig. 13d. The orientation flag shows no indication of particle orientation for this case. The VLDR values are 0.1 for the dust layer and 0.003 at aerosol-free heights of $5-6 \mathrm{~km}$ (not shown here), in accordance to the value of 0.0035 provided in literature for molecular atmospheres (Freudenthaler et al., 2018).

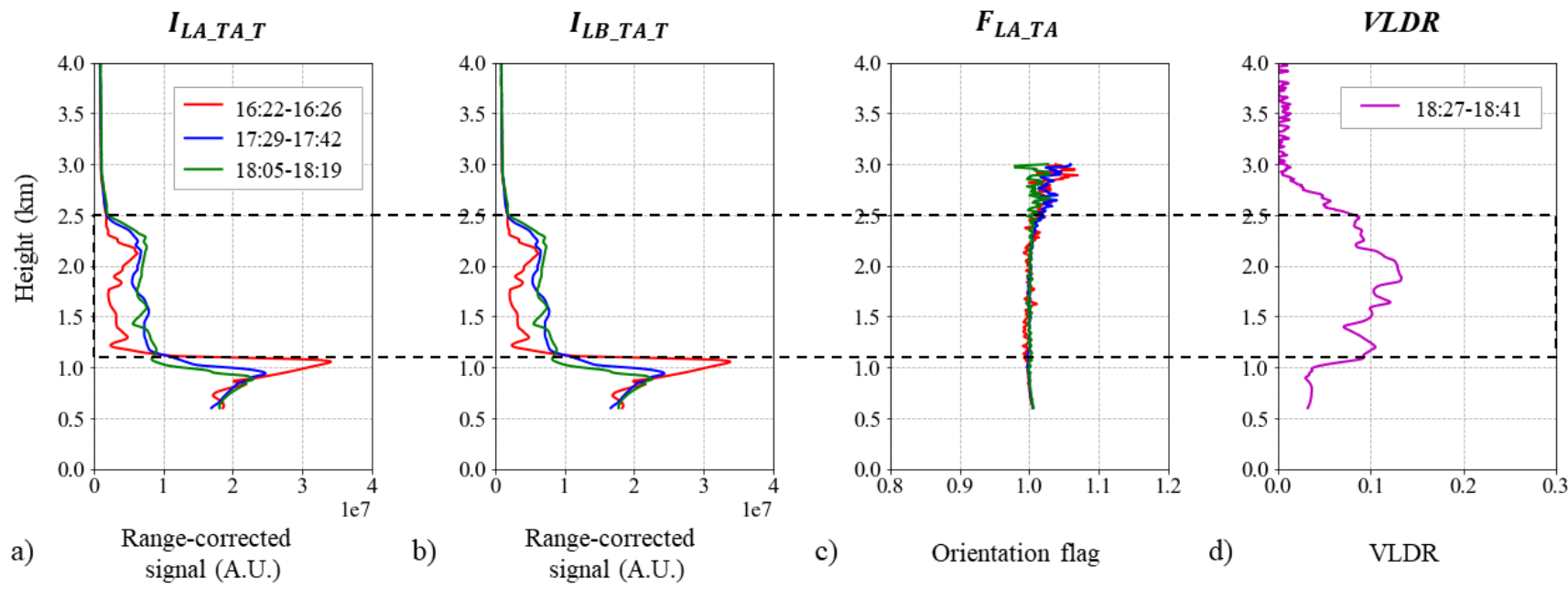

Figure 13. Lidar measurements at $1064 \mathrm{~nm}$ during a dust case at Athens, Greece, on 16 November 2020. The legend shows the time range in UTC for the 15-min averaged signals: a) $I_{L A_{-} T A_{-} T}$, b) $I_{L B_{-} T A_{-} T}$ and c) $F_{L A_{-} T A}$. d) The VLDR measurements at $18: 27-18: 41$ UTC. The heights are above the surface level. 


\section{Overview and future perspectives}

The new polarization lidar nicknamed WALL-E is designed to monitor possible dust particle orientation in the Earth's atmosphere. This work describes in detail the conceptual design, its mechanical and optical parts, the calibration procedures, and finally the first measurements of the system.

The design extends the boundaries of lidar polarimetry, considering the various states of polarization emitted and detected by the system, their interleaved emission and acquisition, which enables the detection of eight signals with two lasers/two telescopes/four detectors. Moreover, an important part of the design is the development of new methodologies for the calibration of the measurements and their alignment with the horizon, so as to define a reference system for the particle orientation.

The mechanical developments include the compact design of the system, its mobility, its ability to perform measurements at the field under a wide range of ambient conditions, and its ability to perform measurements at various zenith and azimuth viewing angles.

Finally, the first measurements are shown for a dust plume on November 162020 in Athens, Greece. The overlap height is at $600 \mathrm{~m}$ and the VLDR measurements reproduce the molecular VLDR in aerosol-free heights, as this is provided in the literature.

The future perspectives of this work, on the technical level, include the modification of the design to provide measurements at further ranges, so as to study particle orientation for desert dust at higher altitudes as well as ice crystals in cirrus clouds. With respect to the analysis of the measurements, we plan to develop a retrieval algorithm that will utilize the measurements from the linearly-polarized and elliptically-polarized emitted light and it will provide not only flags of particle orientation, but also the particle orientation angle, the percentage of oriented particles in the atmosphere and estimations of particle size and refractive index.

The novel two-laser/two-telescope configuration utilized here, has been also implemented on the recently developed EVE lidar system (Paschou et al., 2021). The system was developed to provide ground reference measurements for the validation, assessment and improvement of the L2A products of the Aeolus mission of the European Space Agency (ESA), by deploying the traditional polarization lidar system with linearly polarized emission at $355 \mathrm{~nm}$ on the one hand, and the operation of Aeolus that relies on the circularly polarized emission at $355 \mathrm{~nm}$ on the other.

The detection and monitoring of dust particle orientation in the Earth's atmosphere will unlock our ability for realistic simulations of the desert dust radiative impact, and optimize parameterizations for the natural aerosol component in Earth System Models, in front of today's challenges posed by climate change. The applications of the new polarization lidar do not limit on this work but are anticipated to open new horizons for atmospheric remote sensing. 
https://doi.org/10.5194/amt-2021-30

Preprint. Discussion started: 15 February 2021

(c) Author(s) 2021. CC BY 4.0 License.

\section{Appendix A: Formulas of lidar signals $I_{i \_k \_S}$}

Equations A1-A6 show the formulation of Eq. 7 for the signals $I_{i_{-} k_{-} S}$, taking into account all the optical elements of the system, along with the rotation of the detection units after telescopes $\mathrm{A}$ and $\mathrm{B}$, with respect to the frame coordinate system (Fig. 8). The analytic derivations of Eq. A1-A6 are provided in Section S2 of the Supplement.

\section{A1 I I A_TA_S}

$$
\begin{aligned}
& I_{L A \_T A_{-} S}=E_{L A \_T A} \eta_{S_{-} T A} e \mathbf{M}_{S_{-} T A} \mathbf{M}_{H W_{-} T A} \mathbf{M}_{O_{-} T A} \mathbf{R}_{T A}[\mathbf{F}(\boldsymbol{x})+\mathbf{G}] \boldsymbol{i}_{L A} \Rightarrow
\end{aligned}
$$

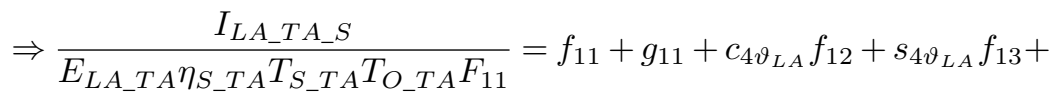

$$
\begin{aligned}
& +D_{S_{-} T A} c_{\left(4 \theta_{T A}+2 \omega_{T A}\right)}\left[f_{12}+c_{4 \vartheta_{L A}}\left(f_{22}+g_{22}\right)+s_{4 \vartheta_{L A}} f_{23}\right]+ \\
& +D_{S_{-} T A} s_{\left(4 \theta_{T A}+2 \omega_{T A}\right)}\left[-f_{13}-c_{4 \vartheta_{L A}} f_{23}+s_{4 \vartheta_{L A}}\left(f_{33}+g_{33}\right)\right]
\end{aligned}
$$

After correcting $I_{L A \_T A \_S}$ for the rotation of the detection unit after telescope A, by setting the fast-axis-angle of $H W P_{T A}$ at $\theta_{T A}=-\frac{\omega_{T A}}{2}$ (Section 4.1), Eq. A1 is written as Eq. A2.

$$
\frac{I_{L A \_} T A_{-} S_{-}\left(\theta_{T A}=-\frac{\omega_{T A}}{2}\right)}{E_{L A \_T A} \eta_{S_{-} T A} T_{S_{-} T A} T_{O_{-} T A} F_{11}}=f_{11}+g_{11}+c_{4 \vartheta_{L A}} f_{12}+s_{4 \vartheta_{L A}} f_{13}+D_{S_{-} T A}\left[f_{12}+c_{4 \vartheta_{L A}}\left(f_{22}+g_{22}\right)+s_{4 \vartheta_{L A}} f_{23}\right]
$$

\section{A2 $I_{L B \_T A \_S}$}

$$
\begin{aligned}
I_{L B_{-} T A_{-} S}=E_{L_{-} T A} \eta_{S_{-} T A} \boldsymbol{e} \mathbf{M}_{S_{-} T A} & \mathbf{M}_{H_{W_{-}} T A} \mathbf{M}_{O_{-} T A} \mathbf{R}_{T A}[\mathbf{F}(\boldsymbol{x})+\mathbf{G}] \boldsymbol{i}_{L B} \Rightarrow \\
\Rightarrow \frac{I_{L B_{-} T A \_}}{E_{L B_{-} T A} \eta_{S_{-} T A} T_{S_{-} T A} T_{O_{-} T A} F_{11}}= & f_{11}+\left[D_{S_{-} T A} c_{\left(4 \theta_{T A}+2 \omega_{T A}\right)}+c_{2 \varphi_{L B}} c_{\left(4 \theta_{L B}-2 \phi_{L B}\right)}\right] f_{12}+ \\
& +\left[c_{2 \varphi_{L B}} s_{\left(4 \theta_{L B}-2 \phi_{L B}\right)}-D_{S_{-} T A} s_{\left(4 \theta_{T A}+2 \omega_{T A}\right)}\right] f_{13} \\
& -s_{2 \varphi_{L B}} f_{14}+D_{S_{-} T A} c_{\left(4 \theta_{T A}+2 \omega_{T A}\right)} c_{2 \varphi_{L B}} c_{\left(4 \theta_{L B}-2 \phi_{L B}\right)} f_{22}+ \\
& +D_{S_{-} T A} c_{2 \varphi_{L B}}\left[c_{\left(4 \theta_{T A}+2 \omega_{T A}\right)} s_{\left(4 \theta_{L B}-2 \phi_{L B}\right)}-s_{\left(4 \theta_{T A}+2 \omega_{T A}\right)} c_{\left(4 \theta_{L B}-2 \phi_{L B}\right)}\right] f_{23}+ \\
& -D_{S_{-} T A} c_{\left(4 \theta_{T A}+2 \omega_{T A}\right)} s_{2 \varphi_{L B}} f_{24}+D_{S_{-} T A} s_{\left(4 \theta_{T A}+2 \omega_{T A}\right)} c_{2 \varphi_{L B}} s_{\left(4 \theta_{L B}-2 \phi_{L B}\right)} f_{33}+ \\
& -D_{S_{-} T A} s_{\left(4 \theta_{T A}+2 \omega_{T A}\right)} s_{2 \varphi_{L B}} f_{34}+ \\
& +g_{11}+D_{S_{-} T A} c_{\left(4 \theta_{T A}+2 \omega_{T A}\right)} c_{2 \varphi_{L B}} c_{\left(4 \theta_{L B}-2 \phi_{L B}\right)} g_{22}+ \\
& +D_{S_{-} T A} s_{\left(4 \theta_{T A}+2 \omega_{T A}\right)} c_{2 \varphi_{L B}} s_{\left(4 \theta_{L B}-2 \phi_{L B}\right)} g_{33}
\end{aligned}
$$


https://doi.org/10.5194/amt-2021-30

Preprint. Discussion started: 15 February 2021

(c) Author(s) 2021. CC BY 4.0 License.

(c) (1)
Atmospheric Measurement

Techniques

Discussions

After correcting $I_{L B_{-} T A_{-} S}$ for the rotation of the detection unit after telescope A, by setting the fast-axis-angle of $H W P_{T A}$ at $\theta_{T A}=-\frac{\omega_{T A}}{2}$ (Section 4.1), Eq. A3 is written as Eq. A4.

$$
\begin{aligned}
\frac{I_{L B_{-} T A_{-} S_{-}\left(\theta_{T A}=-\frac{\omega_{T A}}{2}\right)} T_{L B_{-} T A} \eta_{S_{-} T A} T_{S_{-} T A} T_{O_{-} T A} F_{11}}{E_{11}} & f_{11}+\left[D_{S_{-} T A}+c_{2 \varphi_{L B}} c_{\left(4 \theta_{L B}-2 \phi_{L B}\right)}\right] f_{12}+c_{2 \varphi_{L B}} s_{\left(4 \theta_{L B}-2 \phi_{L B}\right)} f_{13}-s_{2 \varphi_{L B}} f_{14}+ \\
& +D_{S_{-} T A} c_{2 \varphi_{L B}} c_{\left(4 \theta_{L B}-2 \phi_{L B}\right)} f_{22}+D_{S_{-} T A} c_{2 \varphi_{L B}} s_{\left(4 \theta_{L B}-2 \phi_{L B}\right)} f_{23}-D_{S_{-} T A} s_{2 \varphi_{L B}} f_{24}+ \\
& +g_{11}+D_{S_{-} T A} c_{2 \varphi_{L B}} c_{\left(4 \theta_{L B}-2 \phi_{L B}\right)} g_{22}
\end{aligned}
$$

\section{A3 $I_{L A} A_{-} B_{-} S$}

$I_{L A \_T B \_S}=E_{L A \_T B} \eta_{S \_T B} e \mathbf{M}_{S_{-} T B} \mathbf{M}_{Q W_{-} T B} \mathbf{M}_{H W_{-} T B} \mathbf{M}_{O_{-} T B} \mathbf{R}_{T B}[\mathbf{F}(\boldsymbol{x})+\mathbf{G}] \boldsymbol{i}_{L A} \Rightarrow$

$\Rightarrow \frac{I_{L A_{-} T B_{-} S}}{E_{L A \_T B} \eta_{S_{-} T B} T_{S_{-} T B} T_{O_{-} T B} F_{11}}=f_{11}+c_{4 \vartheta_{L A}} f_{12}+s_{4 \vartheta_{L A}} f_{13}+D_{S_{-} T B} f_{14}+D_{S_{-} T B} c_{4 \vartheta_{L A}} f_{24}-D_{S_{-} T B} s_{4 \vartheta_{L A}} f_{34}+g_{11}$

\section{A4 $I_{L B_{-} T B_{-} S}$}

$I_{L B_{-} T B \_S}=E_{L B_{-} T B} \eta_{S_{-} T B} \boldsymbol{e} \mathbf{M}_{S_{-} T B} \mathbf{M}_{Q W_{-} T B} \mathbf{M}_{H W_{-} T B} \mathbf{M}_{O_{-} T B} \mathbf{R}_{T B}[\mathbf{F}(\boldsymbol{x})+\mathbf{G}] \boldsymbol{i}_{L B} \Rightarrow$

$$
\begin{aligned}
& \Rightarrow \frac{I_{L B_{-} T B_{-} S}}{E_{L B_{-} T B} \eta_{S_{-} T B} T_{S_{-} T B} T_{O_{-} T B} F_{11}}=f_{11}+c_{2 \varphi_{L B}} c_{\left(4 \theta_{L B}-2 \phi_{L B}\right)} f_{12}+c_{2 \varphi_{L B}} s_{\left(4 \theta_{L B}-2 \phi_{L B}\right)} f_{13}+\left[D_{S_{-} T B}-s_{2 \varphi_{L B}}\right] f_{14}+ \\
& +D_{S_{-} T B} c_{2 \varphi_{L B}} c_{\left(4 \theta_{L B}-2 \phi_{L B}\right)} f_{24}-D_{S_{-} T B} c_{2 \varphi_{L B}} s_{\left(4 \theta_{L B}-2 \phi_{L B}\right)} f_{34}-D_{S_{-} T B} s_{2 \varphi_{L B}} f_{44}+ \\
& +g_{11}-D_{S_{-} T B} s_{2 \varphi_{L B}} g_{44}
\end{aligned}
$$




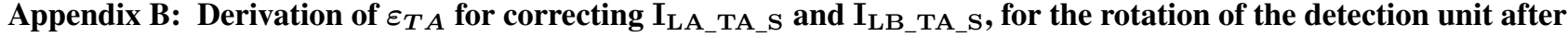
telescope A.

After placing a linear polarizer in front of the window of laser A at $45^{\circ}$ from $x_{F}$-axis (Fig. 9), we acquire the measurements

$I_{L A \_T A \_S \_45^{\circ}}$ (Eq. S28 in the Supplement). Considering $\theta_{T A}=\frac{-\omega_{T A}}{2}+\varepsilon_{T A}$ we derive $I_{L A \_T A_{-} S_{-} 45^{\circ} \_}\left(\theta_{T A}=-\frac{\omega_{T A}}{2}+\varepsilon_{T A}\right)$ as shown in Eq. B1.

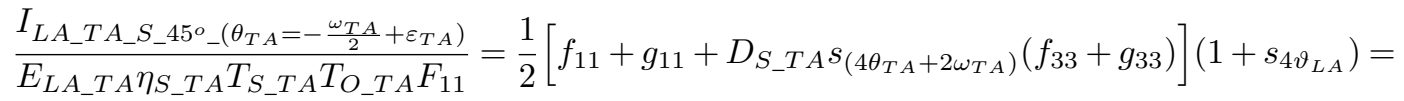

$$
\begin{aligned}
& =\frac{1}{2}\left[f_{11}+g_{11}+D_{S_{-} T A} s_{\left(4\left(-\frac{\omega_{T A}}{2}+\varepsilon_{T A}\right)+2 \omega_{T A}\right)}\left(f_{33}+g_{33}\right)\right]\left(1+s_{4 \vartheta_{L A}}\right)= \\
& =\frac{1}{2}\left[f_{11}+g_{11}+D_{S_{-} T A} s_{4 \varepsilon_{T A}}\left(f_{33}+g_{33}\right)\right]\left(1+s_{4 \vartheta_{L A}}\right) \Rightarrow \\
& \Rightarrow \frac{I_{L A \_T A_{-} S_{-} 45^{\circ} \_}\left(\theta_{T A}=-\frac{\omega_{T A}}{2}+\varepsilon_{T A}\right)}{E_{L A \_T A} \eta_{S \_T A} T_{S \_T A} T_{O_{-} T A} F_{11}\left(f_{11}+g_{11}\right)}=\frac{1}{2}\left[1-a D_{S \_T A} s_{4 \varepsilon_{T A}}\right]\left(1+s_{4 \vartheta_{L A}}\right)
\end{aligned}
$$

$a$ is the atmospheric polarization parameter with $a=\frac{f_{22}+g_{22}}{f_{11}+g_{11}}=-\frac{f_{33}+g_{33}}{f_{11}+g_{11}}$.

We derive $\varepsilon_{T A}$ in a similar way as the " $\Delta 90^{\circ}$ calibration" (Section 11 in Freudenthaler (2016)), by rotating the $H W P_{T A}$ by an additional angle of $0^{\circ}$ and $45^{\circ}$ with respect to the $x_{D U_{T A}}$-axis (Fig. 10). The respective calculations are provided in Eq. B2-B9:

$$
\begin{aligned}
\eta^{*}\left(\theta_{T A}=+45^{o}-\frac{\omega_{T A}}{2}+\varepsilon_{T A}\right) & =\frac{I_{L A \_} T A_{-} R_{-} 45^{\circ} \_\left(\theta_{T A}=45-\frac{\omega_{T A}}{2}+\varepsilon_{T A}\right)}{\left.I_{L A \_T A_{-} T_{-} 45^{\circ}\left(\theta_{T A}=45-\frac{\omega_{T A}}{2}+\varepsilon_{T A}\right)}\right)}=\eta_{T A} \frac{\left(1+a s_{4\left(45+\varepsilon_{T A}\right)}\right)\left(1+s_{4 \vartheta_{L A}}\right)}{\left(1-a s_{4\left(45+\varepsilon_{T A}\right)}\right)\left(1+s_{4 \vartheta_{L A}}\right)}= \\
& =\eta_{T A} \frac{1-a s_{4 \varepsilon_{T A}}}{1+a s_{4 \varepsilon_{T A}}}
\end{aligned}
$$

$Y\left(\varepsilon_{T A}, a\right)=\frac{\eta^{*}\left(\theta_{T A}=-\frac{\omega_{T A}}{2}+\varepsilon_{T A}\right)-\eta^{*}\left(\theta_{T A}=+45^{o}-\frac{\omega_{T A}}{2}+\varepsilon_{T A}\right)}{\eta^{*}\left(\theta_{T A}=-\frac{\omega_{T A}}{2}+\varepsilon_{T A}\right)+\eta^{*}\left(\theta_{T A}=+45^{o}-\frac{\omega_{T A}}{2}+\varepsilon_{T A}\right)}=\frac{2 a s_{4 \varepsilon_{T A}}}{1+a^{2} s_{4 \varepsilon_{T A}}^{2}}$ 
Following the tangent half-angle substitution (Section S.12.1 in Freudenthaler (2016)) we derive $\varepsilon_{T A}$ and $a$ by successive approximation, as shown in Eq. B5-B9.

$390 \varepsilon_{T A}=\frac{1}{4} \arcsin \left[\frac{1}{a} \tan \left(\frac{\arcsin \left(Y\left(\varepsilon_{T A}, a\right)\right)}{2}\right)\right]$

$a=\frac{1}{s_{4 \varepsilon_{T A}}} \tan \left(\frac{\arcsin \left(Y\left(\varepsilon_{T A}, a\right)\right)}{2}\right)$

As a first approximation of $\varepsilon_{T A}$ we calculate $\varepsilon_{T A_{l}}$ with Eq. B7.

$\varepsilon_{T A_{l}}=\frac{1}{4} \arcsin \left[\tan \left(\frac{\arcsin \left(Y\left(\varepsilon_{T A}, a\right)\right)}{2}\right)\right]<\varepsilon_{T A}$

After adjusting the $H W P_{T A}$ by $-\varepsilon_{T A_{l}}$, which results in $\theta_{T A}=-\frac{\omega_{T A}}{2}+\varepsilon_{T A}-\varepsilon_{T A_{l}}$, we derive $Y\left(\varepsilon_{T A}-\varepsilon_{T A_{l}}, a\right)$ (Eq. B8).

395 Then, $\varepsilon_{T A}$ is calculated by Eq. B9.

$Y\left(\varepsilon-\varepsilon_{T A_{l}}, a\right)=\frac{2 a s_{4\left(\varepsilon-\varepsilon_{T A_{l}}\right)}}{\left.1+a^{2} s_{4\left(\varepsilon-\varepsilon_{T A_{l}}\right.}^{2}\right)}$

$\frac{Y\left(\varepsilon_{T A}-\varepsilon_{T A_{l}}, a\right)}{Y\left(\varepsilon_{T A}, a\right)}=\frac{s_{4\left(\varepsilon_{T A}-\varepsilon_{T A_{l}}\right)}\left(1+s_{4 \varepsilon_{T A}}^{2} a^{2}\right)}{s_{4 \varepsilon_{T A}}\left(1+s_{4\left(\varepsilon_{T A}-\varepsilon_{T A_{l}}\right)}^{2} a^{2}\right)} \approx \frac{s_{4\left(\varepsilon_{T A}-\varepsilon_{T A_{l}}\right)}}{s_{4 \varepsilon_{T A}}} \approx \frac{\varepsilon_{T A}-\varepsilon_{T A_{l}}}{\varepsilon_{T A}}=1-\frac{\varepsilon_{T A_{l}}}{\varepsilon_{T A}} \Rightarrow$

$\Rightarrow \varepsilon_{T A} \approx \frac{Y\left(\varepsilon_{T A}, a\right)}{Y\left(\varepsilon_{T A}, a\right)-Y\left(\varepsilon_{T A}-\varepsilon_{T A_{l}}, a\right)} \varepsilon_{T A_{l}}$

Figure B1 shows the test performed for zeroing $\varepsilon_{T A}$ and setting $\theta_{T A}=\frac{-\omega_{T A}}{2}$ (step 3 in Fig. 10), by the successive steps described above. Specifically, with zero rotation of the $H W P_{T A}, \varepsilon_{T A_{l}}=4.69^{\circ}$, with $-4.69^{\circ}$ rotation of the $H W P_{T A}, \varepsilon_{T A_{l}}=$ $400-0.22^{\circ}$, and finally with $-4.69^{\circ}+0.22^{\circ}=-4.47^{\circ}$ rotation of the $H W P_{T A}, \varepsilon_{T A_{l}}=0^{\circ}$. 


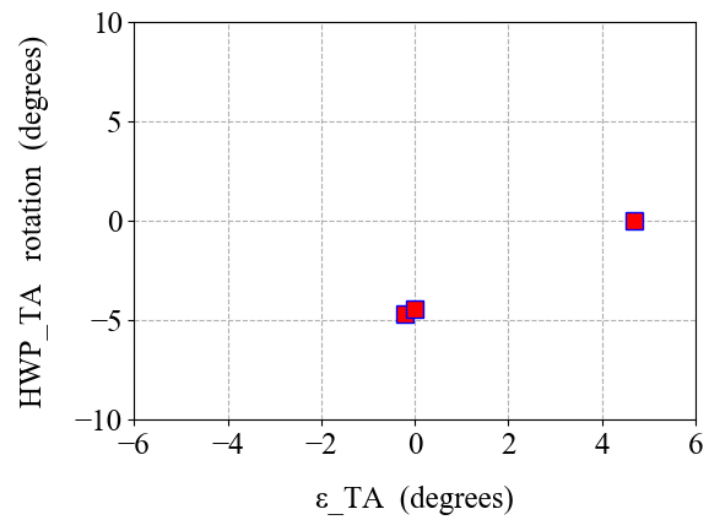

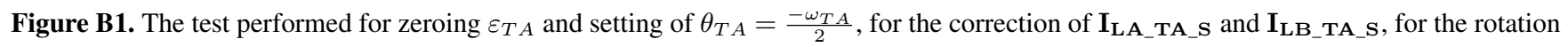
of the detection unit after telescope A.

\section{Appendix C: Derivation of $\varepsilon_{L A}$ for defining the polarization of $i_{L A}$ with respect to the horizon}

For the derivation of the $\varepsilon_{L A}$ we use the signals $I_{L A_{-} T A_{-} S}$ (Eq. A2), after they are corrected for the effect of the rotation of the detection unit after telescope A, by setting $\theta_{T A}=-\frac{\omega_{T A}}{2}$ (Section 4.1). Moreover, we consider that the atmosphere consists of randomly-oriented particles, thus the off-diagonal elements in Eq. A2 are zero. Then, the signals $I_{L A_{-} T A_{-} S\left(\theta_{T A}=-\frac{\omega_{T A}}{2}\right)}$ are provided in Eq. $\mathrm{C} 1$.

$$
\begin{aligned}
I_{L A \_T A \_} S\left(\theta_{T A}=-\frac{\omega_{T A}}{2}\right) & =E_{L A \_T A} \eta_{S_{-} T A} T_{S_{-} T A} T_{O_{-} T A} F_{11}\left[f_{11}+g_{11}+D_{S_{-} T A} c_{4 \vartheta_{L A}}\left(f_{22}+g_{22}\right)\right]= \\
& =E_{L A \_T A} \eta_{S_{-} T A} T_{S_{-} T A} T_{O_{-} T A} F_{11}\left(f_{11}+g_{11}\right)\left[1+a D_{S_{-} T A} c_{4 \vartheta_{L A}}\right]
\end{aligned}
$$

Where, $\vartheta_{L A}=\varepsilon_{L A}$ and $a$ is the atmospheric polarization parameter, $a=\frac{f_{22}+g_{22}}{f_{11}+g_{11}}$.

We rotate the $H W P_{L A}$ by $+22.5^{\circ}$ and $-22.5^{\circ}$ with respect to the $x_{F}$-axis and we derive $\varepsilon_{L A}$ by performing the " $\Delta 90^{\circ}$ calibration" of Freudenthaler (2016), as shown in Eq. C2-C8.

$$
\eta^{*}\left(\vartheta_{L A}=+22.5^{\circ}+\varepsilon_{L A}\right)=\frac{I_{L A_{-} T A_{-} R\left(\theta_{T A}=-\frac{\omega_{T A}}{2}\right)_{-}\left(\vartheta_{L A}=22.5+\varepsilon_{L A}\right)}}{I_{L A \_} T A_{-} T\left(\theta_{T A}=-\frac{\omega_{T A}}{2}\right)_{-}\left(\vartheta_{L A}=22.5+\varepsilon_{L A}\right)}=\eta_{T A} \frac{1-a c_{4\left(22.5+\varepsilon_{L A}\right)}}{1+a c_{4\left(22.5+\varepsilon_{L A}\right)}}=
$$

$$
=\eta_{T A} \frac{1-a c_{\left(90+4 \varepsilon_{L A}\right)}}{1+a c_{\left(90+4 \varepsilon_{L A}\right)}}=\eta_{T A} \frac{1+a s_{4 \varepsilon_{L A}}}{1-a s_{4 \varepsilon_{L A}}}
$$




$$
\begin{aligned}
\eta^{*}\left(\vartheta_{L A}=-22.5^{o}+\varepsilon_{L A}\right) & =\frac{I_{L A_{-} T A_{-} R\left(\theta_{T A}=-\frac{\omega_{T A}}{2}\right)_{-}\left(\vartheta_{L A}=-22.5+\varepsilon_{L A}\right)}}{I_{L A_{-} T A_{-} T\left(\theta_{T A}=-\frac{\omega_{T A}}{2}\right)_{-}\left(\vartheta_{L A}=-22.5+\varepsilon_{L A}\right)}}=\eta_{T A} \frac{1-a c_{\left(-90+4 \varepsilon_{L A}\right)}}{1+a c_{\left(-90+4 \varepsilon_{L A}\right)}}= \\
& =\eta_{T A} \frac{1-a s_{4 \varepsilon_{L A}}}{1+a s_{4 \varepsilon_{L A}}}
\end{aligned}
$$

$Y\left(\varepsilon_{L A}, a\right)=\frac{\eta^{*}\left(\vartheta_{L A}=+22.5^{o}+\varepsilon_{L A}\right)-\eta^{*}\left(\vartheta_{L A}=-22.5^{o}+\varepsilon_{L A}\right)}{\eta^{*}\left(\vartheta_{L A}=+22.5^{o}+\varepsilon_{L A}\right)+\eta^{*}\left(\vartheta_{L A}=-22.5^{o}+\varepsilon_{L A}\right)}=\frac{2 a s_{4 \varepsilon_{L A}}}{1+a^{2} s_{4 \varepsilon_{L A}}^{2}}$

Following the tangent half-angle substitution (Section S.12.1 in Freudenthaler (2016)) we derive $\varepsilon_{L A}$, as shown in Eq. C5-C8.

$415 \varepsilon_{L A}=\frac{1}{4} \arcsin \left[\frac{1}{a} \tan \left(\frac{\arcsin \left(Y\left(\varepsilon_{L A}, a\right)\right)}{2}\right)\right]$

As a first approximation of $\varepsilon_{L A}$ we calculate $\varepsilon_{L A_{l}}$ with Eq. C6.

$\varepsilon_{L A_{l}}=\frac{1}{4} \arcsin \left[\tan \left(\frac{\arcsin \left(Y\left(\varepsilon_{L A}, a\right)\right)}{2}\right)\right]<\varepsilon_{L A}$

After adjusting the $H W P_{L A}$ by $-\varepsilon_{L A_{l}}$, which results in $\vartheta_{L A}=\varepsilon_{L A}-\varepsilon_{L A_{l}}$ with respect to the $x_{F}$-axis, we derive $Y\left(\varepsilon_{L A}-\right.$ $\left.\varepsilon_{L A_{l}}, a\right)$ (Eq. C7). Then, $\varepsilon_{L A}$ is calculated by Eq. C8.

$Y\left(\varepsilon_{L A}-\varepsilon_{L A_{l}}, a\right)=\frac{2 a s_{4\left(\varepsilon_{L A}-\varepsilon_{L A_{l}}\right)}}{1+a^{2} s_{4\left(\varepsilon_{L A}-\varepsilon_{L A_{l}}\right)}^{2}}$

$\frac{Y\left(\varepsilon_{L A}-\varepsilon_{L A_{l}}, a\right)}{Y\left(\varepsilon_{L A}, a\right)}=\frac{s_{4\left(\varepsilon_{L A}-\varepsilon_{L A_{l}}\right)}\left(1+s_{4 \varepsilon_{L A}}^{2} a^{2}\right)}{s_{4 \varepsilon_{L A}}\left(1+s_{4\left(\varepsilon_{L A}-\varepsilon_{L A_{l}}\right)}^{2} a^{2}\right)} \approx \frac{s_{4\left(\varepsilon_{L A}-\varepsilon_{L A_{l}}\right)}}{s_{4 \varepsilon_{L A}}} \approx \frac{\varepsilon_{L A}-\varepsilon_{L A_{l}}}{\varepsilon_{L A}}=1-\frac{\varepsilon_{L A_{l}}}{\varepsilon_{L A}} \Rightarrow$

$\Rightarrow \varepsilon_{L A} \approx \frac{Y\left(\varepsilon_{L A}, a\right)}{Y\left(\varepsilon_{L A}, a\right)-Y\left(\varepsilon_{L A}-\varepsilon_{L A_{l}}, a\right)} \varepsilon_{L A_{l}}$

Figure $\mathrm{C} 1$ shows the test performed for zeroing $\varepsilon_{L A}$ and setting $\vartheta_{L A}=22.5^{\circ}$ (step 2 in Fig. 11), by the successive steps described above. Specifically, with zero rotation of the $H W P_{L A}, \varepsilon_{L A_{l}}=4.03^{\circ}$, with $-4.03^{\circ}$ rotation of the $H W P_{L A}, \varepsilon_{L A_{l}}=$ 
$0.56^{\circ}$, with $-4.03^{\circ}-0.56^{\circ}=-4.59^{\circ}$ rotation of the $H W P_{L A}, \varepsilon_{L A_{l}}=0.13^{\circ}$, and finally with $-4.59^{\circ}-0.13^{\circ}=-4.72^{\circ}$ rotation of the $H W P_{L A}, \varepsilon_{L A_{l}}=0^{\circ}$.

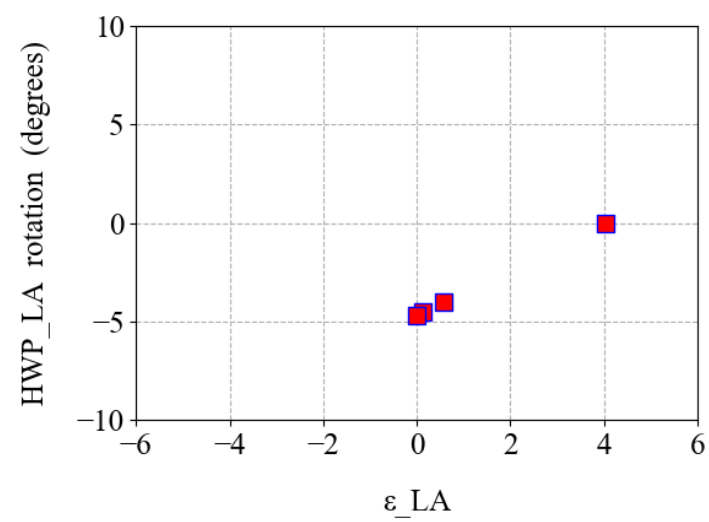

Figure C1. The test performed for zeroing $\varepsilon_{L A}$, for defining the polarization of $\boldsymbol{i}_{L A}$ with respect to the horizon, by setting the angle of polarization at $2 \vartheta_{L A}=45^{\circ}$.

\section{Appendix D: Derivation of the angle of the polarization ellipse $\alpha$ and of the degree of linear polarization $b$, for defining the polarization of $i_{L B}$ with respect to the horizon.}

\section{D1 Derivation of the angle of the polarization ellipse $\alpha$}

The signals $I_{L B \_T A \_S}$ (Eq. A4) are provided by Eq. D1, considering an atmosphere with randomly-oriented particles (all offdiagonal elements of the backscatter matrix are zero), and that the detection unit after telescope A is aligned with the system frame (Section 4.1). Note that in Eq. D1 " $a$ " is the atmospheric polarization parameter, whereas " $\alpha "$ is the the angle of the polarization ellipse of the light from the emission unit of laser $\mathrm{B}$.

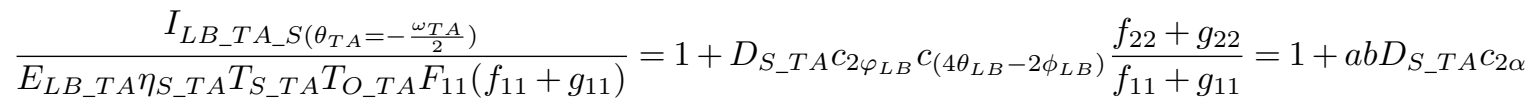


In order to derive the angle $\alpha$ we rotate the $H W P_{L B}$ by $+22.5^{\circ}$ and $-22.5^{\circ}$ with respect to the $x_{F}$-axis, so as $\alpha_{+45^{\circ}}=$ $\alpha+45^{\circ}$ and $\alpha_{-45^{\circ}}=\alpha-45^{\circ}$, respectively (since $\alpha=2 \theta_{L B}-\phi_{L B}$, Eq. 19). We then perform a methodology similar to the " $\Delta 90^{\circ}$ calibration" of Freudenthaler (2016) to derive $\alpha$, as shown in Eq. D2-D8.

$\eta^{*}\left(\alpha_{+45^{\circ}}=\alpha+45^{\circ}\right)=\frac{I_{L B_{-} T A_{-} R\left(\theta_{T A}=-\frac{\omega_{T A}}{2}\right)_{\_} \alpha_{+45^{\circ}}}}{I_{L B_{-} T A_{-} T\left(\theta_{T A}=-\frac{\omega_{T A}}{2}\right)_{-} \alpha_{+45^{\circ}}}}=\eta_{T A} \frac{1-a b c_{(2 \alpha+90)}}{1+a b c_{(2 \alpha+90)}}=\eta_{T A} \frac{1+a b s_{2 \alpha}}{1-a b s_{2 \alpha}}$

$\eta^{*}\left(\alpha_{-45^{\circ}}=\alpha-45^{\circ}\right)=\frac{I_{L B_{-} T A_{-} R\left(\theta_{T A}=-\frac{\omega_{T A}}{2}\right)_{-} \alpha_{-45^{\circ}}}}{I_{L B_{-} T A_{-} T\left(\theta_{T A}=-\frac{\omega_{T A A}}{2}\right)_{-} \alpha_{-45^{\circ}}}}=\eta_{T A} \frac{1-a b c_{(2 \alpha-90)}}{1+a b c_{(2 \alpha-90)}}=\eta_{T A} \frac{1-a b s_{2 \alpha}}{1+a b s_{2 \alpha}}$

$Y(\alpha, a, b)=\frac{\eta^{*}\left(\alpha_{+45^{\circ}}=\alpha+45^{o}\right)-\eta^{*}\left(\alpha_{-45^{\circ}}=\alpha-45^{o}\right)}{\eta^{*}\left(\alpha_{+45^{\circ}}=\alpha+45^{o}\right)+\eta^{*}\left(\alpha_{-45^{o}}=\alpha-45^{o}\right)}=\frac{2 a b s_{2 \alpha}}{1+a^{2} b^{2} s_{2 \alpha}^{2}}$

Following the tangent half-angle substitution (Section S.12.1 in Freudenthaler (2016)) we derive $\alpha$ by successive approximation, as shown in Eq. D5-D9.

$\alpha=\frac{1}{2} \arcsin \left[\frac{1}{a b} \tan \left(\frac{\arcsin (Y(\alpha, a, b))}{2}\right)\right]$

As a first approximation of $\alpha$ we calculate $\alpha_{l}$ with Eq. D6.

$\alpha_{l}=\frac{1}{2} \arcsin \left[\tan \left(\frac{\arcsin (Y(\alpha, a, b))}{2}\right)\right]$

After adjusting the $H W P_{L B}$ by $-\frac{\alpha_{l}}{2}$, which results in $\alpha_{n e w}=\alpha-\alpha_{l}$ (Eq. 19), we derive $Y\left(\alpha-\alpha_{l}, a, b\right)$ (Eq. D7). Then, $\alpha$ is calculated by Eq. D8.

$Y\left(\alpha-\alpha_{l}, a, b\right)=\frac{\eta^{*}\left(+45^{o}+\alpha-\alpha_{l}\right)-\eta^{*}\left(-45^{o}+\alpha-\alpha_{l}\right)}{\eta^{*}\left(+45^{o}+\alpha-\alpha_{l}\right)+\eta^{*}\left(-45^{o}+\alpha-\alpha_{l}\right)}==\frac{2 a b s_{2\left(\alpha-\alpha_{l}\right)}}{1+a^{2} b^{2} s_{2\left(\alpha-\alpha_{l}\right)}^{2}}$

$$
\begin{aligned}
& \frac{Y\left(\alpha-\alpha_{l}, a, b\right)}{Y(\alpha, a, b)}=\frac{s_{2\left(\alpha-\alpha_{l}\right)}\left(1+a^{2} b^{2} s_{2 \alpha}^{2}\right)}{s_{2 \alpha}\left(1+a^{2} b^{2} s_{2\left(\alpha-\alpha_{l}\right)}^{2}\right)} \approx \frac{\alpha-\alpha_{l}}{\alpha}=1-\frac{\alpha_{l}}{\alpha} \Rightarrow \\
& \Rightarrow \alpha \approx \frac{Y(\alpha, a, b)}{Y(\alpha, a, b)-Y\left(\alpha-\alpha_{l}, a, b\right)} \alpha_{l}
\end{aligned}
$$




\section{D2 Derivation of the degree of linear polarization $b$}

450 As shown in Fig. 12, after calculating $\alpha$ in Section D1, we rotate the $H W P_{L B}$ by $-\frac{\alpha}{2}$ and by $22.5^{\circ}-\frac{\alpha}{2}$, so as $\alpha_{1}=0^{\circ}$ and $\alpha_{2}=45^{\circ}$ with respect to the $x_{F}$-axis, respectively (Eq. 19).

Then, $b$ is derived from Eq. D9-D11 (using Eq. D1), considering that we have already derived the atmospheric polarization parameter $a$ using the measurements of laser A at the detection unit after telescope A, as shown in Section 6.

$\eta^{*}\left(\alpha_{1}=0^{o}\right)=\frac{I_{L B \_} T A_{-} R\left(\theta_{T A}=-\frac{\omega_{T A}}{2}\right) \_\alpha_{1}}{I_{L B \_} T A_{-} T\left(\theta_{T A}=-\frac{\omega_{T A}}{2}\right)_{-} \alpha_{1}}=\eta_{T A} \frac{1-a b c_{0}}{1+a b c_{0}}=\eta_{T A} \frac{1-a b}{1+a b}$

$455 \quad \eta^{*}\left(\alpha_{2}=+45^{\circ}\right)=\frac{I_{L B_{-} T A \_R\left(\theta_{T A}=-\frac{\omega_{T A}}{2}\right)_{\_} \alpha_{2}}}{I_{L B_{-} T A_{-} T\left(\theta_{T A}=-\frac{\omega_{T A}}{2}\right)_{\_} \alpha_{2}}}=\eta_{T A} \frac{1-a b c_{90}}{1+a b c_{90}}=\eta_{T A}$

$b=\frac{1}{a} \frac{\eta^{*}\left(\alpha_{2}=+45^{o}\right)-\eta^{*}\left(\alpha_{1}=0^{o}\right)}{\eta^{*}\left(\alpha_{2}=+45^{o}\right)+\eta^{*}\left(\alpha_{1}=0^{o}\right)}$

Author contributions. AT and VF formulated the measurement strategy and the emission and detection design of the system, along with the calibration procedures. VF conceived the "two-laser/two-telescope" concept. GG and AL developed the optomechanical design of the instrument. AT and SM tested and optimized the instrument, and acquired the measurements shown herein, with the support of AL, GG, GT, GD, CE and VF. AT, GD and GG performed simulations for defining the capabilities of the system in terms of SNR, JG provided guidance for the scattering calculations used in these simulations and TG provided the corresponding technical support. PP, NS and IB provided software for the quality assurance tests of the measurements. VA supervised and directed the whole project. AT wrote the manuscript and VF, VA, IB, GG, AL, JG and PP provided corrections and suggestions.

Competing interests. No competing interests are present. innovation framework program/ERC grant agreement 725698 (D-TECT). We acknowledge PRACE for awarding us access to MareNostrum at Barcelona Supercomputing Center (BSC), Spain. The work was supported by computational time granted from the Greek Research \& 
https://doi.org/10.5194/amt-2021-30

Preprint. Discussion started: 15 February 2021

(C) Author(s) 2021. CC BY 4.0 License.

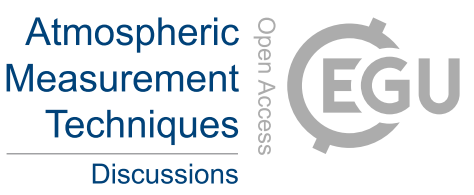

(c) (1)

Discussions

Technology Network (GRNET) in the National HPC facility - ARIS - under project ID pa170906-ADDAPAS, pr005038-REMOD and pr009019-EXEED. Part of the work performed for this study was funded by Romanian National Core Program Contract No.18N/2019 and

470 by the European Regional Development Fund through Competitiveness Operational Programme 2014-2020, POC-A.1-A.1.1.1- F- 2015, project Research Centre for environment and Earth Observation CEO-Terra. 


\section{References}

Balin, Y., Kaul, B., Kokhanenko, G., and Winker, D.: Transformation of light backscattering phase matrices of crystal clouds depending on the zenith sensing angle, Opt. Express 21, 13408-13418, 2013.

Daskalopoulou et al.: Observations of dust particle orientation with the SolPol direct sun polarimeter, Atm. Meas. Tech., 2021 (to be submitted).

Freudenthaler, V.: About the effects of polarising optics on lidar signals and the $\Delta 90$ calibration, Atmos. Meas. Tech., 9, 4181-4255, https://doi.org/10.5194/amt-9-4181-2016, 2016.

Freudenthaler, V., Linné, H., Chaikovski, A., Rabus, D., and Groß, S.: EARLINET lidar quality assurance tools, Atmos. Meas. Tech. Discuss., https://doi.org/10.5194/amt-2017-395, in review, 2018.

Gasteiger, J., Wiegner, M., Groß, S., Freudenthaler, V., Toledano, C., Tesche, M., and Kandler, K.: Modelling lidar-relevant optical properties of complex mineral dust aerosols, Tellus, B 63, 725-741, doi:10.1111/j.1600-0889.2011.00559.x, 2011.

Geier, M. and Arienti, M.: Detection of preferential particle orientation in the atmosphere: Development of an alternative polarization lidar system, J. Quant. Spectrosc. Ra., 149, 16-32, 2014.

Hayman, M., Spuler, S., Morley, B., and VanAndel, J.: Polarization lidar operation for measuring backscatter phase matrices of oriented scatterers, Opt. Express, 20, 29553-29567, 2012.

Kaul, B.V., Samokhvalov, I.V. and Volkov, S.N.: Investigating Particle Orientation in Cirrus Clouds by Measuring Backscattering Phase Matrices with Lidar, Appl. Optics, 43, 6620-6628, 2004.

Kokhanenko, G. P., Balin, Y. S., Klemasheva, M. G., Nasonov, S. V., Novoselov, M. M., Penner, I. E., and Samoilova, S. V.: Scanning polarization lidar LOSA-M3: opportunity for research of crystalline particle orientation in the ice clouds, Atmos. Meas. Tech., 13, 1113-1127, https://doi.org/10.5194/amt-13-1113-2020, 2020.

Paschou, P. et al.: The EVE polarization lidar system for the Aeolus L2A Cal/Val, Atmos. Meas. Tech., 2021, (to be submitted).

Tsekeri, A. et al.: Polarization lidar for detecting dust orientation: Use a novel dust scattering database and machine learning tools for the system design, Atmos. Meas. Tech., 2021, (to be submitted).

Ulanowski, Z., Bailey, J., Lucas, P. W., Hough, J. H., and Hirst, E.: Alignment of atmospheric mineral dust due to electric field, Atmos. Chem. Phys., 7, 6161-6173, https://doi.org/10.5194/acp-7-6161-2007, 2007.

Volkov, S. N., Samokhvalov, I. V., Cheong, H. D., and Kim, D.: Investigation of East Asian clouds with polarization light detection and ranging, Appl. Optics, 54, 3095-3105, 2015.

Whitney, B.A., Wolff, M.J.: Scattering and absorption by aligned grains in circumstellar environments. Astrophys. J. 574, 205-231, 2002. 